\title{
GENERIC INNER PROJECTIONS OF PROJECTIVE VARIETIES AND AN APPLICATION TO THE POSITIVITY OF DOUBLE POINT DIVISORS
}

\begin{abstract}
ATSUSHI NOMA
Abstract. Let $X \subseteq \mathbb{P}^{N}$ be a smooth nondegenerate projective variety of dimension $n \geq 2$, codimension $e$ and degree $d$ with the canonical line bundle $\omega_{X}$ defined over an algebraically closed field of characteristic zero. The purpose here is to prove that the base locus of $\left|\mathcal{O}_{X}(d-n-e-1) \otimes \omega_{X}^{\vee}\right|$ is at most a finite set, except in a few cases. To describe the exceptional cases, we classify (not necessarily smooth) projective varieties whose generic inner projections have exceptional divisors. As applications, we prove the $(d-e)$-regularity of $\mathcal{O}_{X}$, Property $\left(N_{k-d+e}\right)$ for $\mathcal{O}_{X}(k)$, and inequalities for the delta and sectional genera.
\end{abstract}

\section{INTRODUCTION}

We work over an algebraically closed field $\mathbb{k}$ of characteristic zero. Let $X \subseteq \mathbb{P}^{N}$ be a nondegenerate projective variety of dimension $n$, codimension $e$, and degree $d$. Classically it is well known that if $X$ is smooth with canonical line bundle $\omega_{X}$, the linear system $\left|\mathcal{O}_{X}(d-n-2) \otimes \omega_{X}^{\vee}\right|$ is base-point-free (see [2, Proof of Proposition 3.3]). Here $\omega_{X}^{\vee}$ denotes the dual of $\omega_{X}$. Bo Ilic [1] showed that $\left|\mathcal{O}_{X}(d-n-2) \otimes \omega_{X}^{\vee}\right|$ separates two distinct points of $X$ if $X$ is smooth with $e \geq 2$ and if $X$ is not an isomorphic projection of a Roth variety (see the Remark to Theorem 2 for the definition). The purpose here is to show the further positivity of $\mathcal{O}_{X}(d-n-2) \otimes \omega_{X}^{\vee}$ for $n \geq 2$. As applications, we prove the $(d-e)$-regularity of $\mathcal{O}_{X}$ (which gives an evidence of the regularity conjecture; see [3], 9, §4]), Property $\left(N_{k-d+e}\right)$ for $\mathcal{O}_{X}(k)$, and inequalities for the $\Delta$-genus and the sectional genus of $X$.

To state our result, we introduce two definitions about inner projections of projective varieties. A point $v \in \mathbb{P}^{N}$ is said to be a nonbirational center of $X$ if the linear projection $\pi_{v}: \mathbb{P}^{N} \backslash\{v\} \rightarrow \mathbb{P}^{N-1}$ from $v$ induces a nonbirational map of $X$ to its image. By $\mathcal{C}(X)$ we denote the set of all nonbirational centers in the smooth locus $\operatorname{Sm} X$ of $X$ :

$$
\mathcal{C}(X):=\{u \in \operatorname{Sm} X \mid l(X \cap\langle u, x\rangle) \geq 3 \text { for general } x \in X\} .
$$

Here $l(Z)$ is the length of a scheme $Z$ and \langle\rangle denotes the linear span. (See [15] for nonbirational centers and $\mathcal{C}(X)$.)

On the other hand, we define the exceptional set of generic inner projections.

Received by the editors September 28, 2011 and, in revised form, August 23, 2012.

2010 Mathematics Subject Classification. Primary 14N15, 14N05.

Key words and phrases. Linear projection, inner projection, double point divisor, ramification divisor.

The author was partially supported by Grant-in-Aid for Scientific Research, Japan Society for the Promotion of Science. 
Definition (Condition $\left(E_{m}\right)$ ). Let $m$ be an integer with $0<m \leq e-1$. For general points $x_{1}, \ldots, x_{m} \in X$, we define $E_{x_{1}, \ldots, x_{m}}(X)$ to be the closure of

$$
E_{x_{1}, \ldots, x_{m}}^{0}(X):=\left\{z \in X \backslash\left\langle x_{1}, \ldots, x_{m}\right\rangle \mid \operatorname{dim}\left\langle x_{1}, \ldots, x_{m}, z\right\rangle \cap X \geq 1\right\}(\subseteq X)
$$

i.e., $E_{x_{1}, \ldots, x_{m}}(X)$ is the closure of the positive-dimensional fibres of the projection $\pi_{\Lambda, X}: X \backslash \Lambda \rightarrow \mathbb{P}^{N-m}$ from $\Lambda=\left\langle x_{1}, \ldots, x_{m}\right\rangle$ since $\pi_{\Lambda, X}^{-1}\left(\pi_{\Lambda, X}(z)\right)=$ $\left\langle x_{1}, \ldots, x_{m}, z\right\rangle \cap X \backslash \Lambda$ and $\operatorname{dim} X \cap \Lambda=0$. We say Condition $\left(E_{m}\right)$ holds for $X$ (or $X$ satisfies $\left(E_{m}\right)$ ) if

$$
\operatorname{dim} E_{x_{1}, \ldots, x_{m}}(X) \geq n-1 \quad \text { for general points } x_{1}, \ldots, x_{m} \in X .
$$

For convenience, we define $\left(E_{0}\right)$ so that no $X$ satisfies $\left(E_{0}\right)$ i.e., the condition is false.

For example, a scroll over a projective curve satisfies $\left(E_{1}\right)$, and the Veronese surface in $\mathbb{P}^{5}$ satisfies $\left(E_{2}\right)$ but not $\left(E_{1}\right)$. Here, by a scroll over a projective variety $Z$, we mean the birational image of the projective bundle $\mathbb{P}_{Z}(\mathcal{F})$ for some locally free sheaf $\mathcal{F}$ on $Z$ by the morphism defined by some subsystem of $\left|\mathcal{O}_{\mathbb{P}(\mathcal{F})}(1)\right|$.

We make a few remarks for the definition: (1) Clearly, for an integer $m$ with $1 \leq m<e-1$, if $X$ satisfies $\left(E_{m}\right)$, then $X$ satisfies $\left(E_{m+1}\right)$. (2) To assume the inequalities in (0.2) and (0.3) is equivalent to assuming the equalities there. In fact, by the general position lemma (see Lemma 1.2), $\operatorname{dim}\left\langle x_{1}, \ldots, x_{m}, z\right\rangle \cap X \leq 1$ and $\operatorname{dim} E_{x_{1}, \ldots, x_{m}}(X) \leq n-1$ for general points $x_{1}, \ldots, x_{m} \in X$ and for any point $z \in X$. (3) $\left(E_{m}\right)$ is equivalent to the condition for $\pi_{\Lambda, X}: X \backslash \Lambda \rightarrow \mathbb{P}^{N-m}$ to have an exceptional set of codimension one.

With these definitions, our first result is stated as follows.

Theorem 1. Let $X \subseteq \mathbb{P}^{N}$ be a nondegenerate smooth projective variety of dimension $n \geq 2$, codimension $e \geq 2$, and degree $d$. Let $m$ be an integer with $0<m \leq e-1$. Suppose $X$ does not satisfy $\left(E_{m}\right)$. Then the base locus of $\left|\mathcal{O}_{X}(d-m-n-2) \otimes \omega_{X}^{\vee}\right|$ is contained in $\mathcal{C}(X)$ and $\left|\mathcal{O}_{X}(d-m-n-1) \otimes \omega_{X}^{\vee}\right|$ is very ample on $X \backslash \mathcal{C}(X)$. If we assume furthermore that $\operatorname{dim} \mathcal{C}(X) \leq 0$, then $\mathcal{O}_{X}(d-m-n-2) \otimes \omega_{X}^{\vee}$ is semiample (i.e., some positive multiple is generated by global sections) and $\mathcal{O}_{X}(d-m-n-1) \otimes \omega_{X}^{\vee}$ is ample.

To make the assumptions in Theorem 1 clear, next we will classify projective varieties with $\operatorname{dim} \mathcal{C}(X) \geq 1$ and projective varieties with $\left(E_{m}\right)$. The first case is already known: A nondegenerate projective variety $X \subseteq \mathbb{P}^{N}$ is called a birationaldivisor of type $(\mu, 1)$ of a conical rational scroll for some integer $\mu \geq 2$ if $X$ is the birational image $\psi(\tilde{X})$ of an irreducible and reduced divisor $\tilde{X} \in \mid \mathcal{O}_{\mathbf{E}_{\mathcal{E}}^{L}}(\mu) \otimes$ $p^{*} \mathcal{O}_{\mathbb{P}^{1}}(1) \mid$ of the projective bundle $\mathbf{E}_{\mathcal{E}}^{L}:=\mathbb{P}_{\mathbb{P}^{1}}\left(\mathcal{O}_{\mathbb{P}^{1}}^{\oplus 2} \oplus \mathcal{E}\right)$ for some ample bundle $\mathcal{E}$ on $\mathbb{P}^{1}$ of rank $n-1$. Here $\mathcal{O}_{\mathbf{E}_{\mathcal{E}}^{L}}(1)$ is the tautological line bundle, $p: \mathbf{E}_{\mathcal{E}}^{L} \rightarrow \mathbb{P}^{1}$ is the projection, and $\psi: \mathbf{E}_{\mathcal{E}}^{L} \rightarrow \mathbb{P}^{N}$ is the morphism defined by a subsystem of $\left|\mathcal{O}_{\mathbf{E}_{\mathcal{E}}^{L}}(1)\right|$ such that $\psi$ induces a birational morphism onto its image. Clearly, $\psi\left(\mathbf{E}_{\mathcal{E}}^{L}\right) \subseteq \mathbb{P}^{N}$ is the cone over $\psi\left(\mathbb{P}_{\mathbb{P}^{1}}(\mathcal{E})\right)$ with vertex $L:=\psi\left(\mathbb{P}_{\mathbb{P}^{1}}\left(\mathcal{O}_{\mathbb{P}^{1}}^{\oplus 2}\right)\right)$. Note that if a birational-divisor $X$ of a conical rational scroll is smooth, then the induced morphism $\tilde{X} \rightarrow X$ is finite (especially finite along $L$ ) and hence $\tilde{X} \cong X$ (see [16. Proposition 5.1 (Corollary 6.2)]).

Theorem 2 ([16, (6.2)]). Let $X \subseteq \mathbb{P}^{N}$ be a nondegenerate smooth projective variety with codimension $e \geq 2$. Suppose $\operatorname{dim} \mathcal{C}(X) \geq 1$. Then $\operatorname{dim} \mathcal{C}(X)=1$ and $X$ is a birational-divisor of type $(\mu, 1)$ of a conical rational scroll for some $\mu \geq 2$. 
Remark. In [1, a smooth projective variety $X$ is called a Roth variety if $X$ is a birational-divisor of type $(\mu, 1)$ of a conical rational scroll for $\mu \geq 1$ and if $X$ is embedded by the complete linear system $\left|\mathcal{O}_{X(1)}\right|$. If $\mu=1$ in this case, then $X$ is a rational scroll. For a Roth variety $X$, Bo Ilic observed that $\left.\mathcal{O}_{X}(d-n-2) \otimes \omega_{X}^{\vee}\right|_{L}$ is trivial for $L=\psi\left(\mathbb{P}_{\mathbb{P}^{1}}\left(\mathcal{O}_{\mathbb{P}^{1}}^{\oplus 2}\right)\right.$ ) (see Example $6.3(3)$ ).

For the second case, we have the classification of varieties satisfying $\left(E_{m}\right)$ for $1 \leq m \leq e-1$ without assuming the smoothness of $X$, which is the main part of this paper.

Theorem 3. Let $X \subseteq \mathbb{P}^{N}$ be a nondegenerate (not necessarily smooth) projective variety of dimension $n \geq 2$ and codimension $e$.

(1) If $e \geq 2$ and $X$ satisfies $\left(E_{1}\right)$, then $X$ is projectively equivalent to a scroll over a smooth curve. If $X$ is furthermore smooth, then $X$ is projectively equivalent to a scroll $\mathbb{P}_{C}(\mathcal{F}) \subseteq \mathbb{P}^{N}$ for a locally free sheaf $\mathcal{F}$ on a smooth projective curve $C$.

(2) If $e \geq 3$ and $X$ satisfies $\left(E_{2}\right)$ but not $\left(E_{1}\right)$, then $X$ is projectively equivalent to the cone over the Veronese surface $v_{2}\left(\mathbb{P}^{2}\right)\left(\subseteq \mathbb{P}^{5}\right)$ with $(n-3)$-dimensional vertex in $\mathbb{P}^{n+3}$. If $X$ is furthermore smooth, then $X$ is projectively equivalent to $v_{2}\left(\mathbb{P}^{2}\right)$.

(3) Suppose $e \geq 4$. Then for any $m$ with $e-1>m \geq 3$, it does not occur that $X$ satisfies $\left(E_{m}\right)$ but not $\left(E_{m-1}\right)$. Consequently, $X$ satisfies $\left(E_{m}\right)$ for some $m$ with $e-1 \geq m \geq 3$ if and only if $X$ satisfies $\left(E_{1}\right)$.

Here we say that two projective varieties $X \subseteq \mathbb{P}^{N}$ and $X^{\prime} \subseteq \mathbb{P}^{N^{\prime}}$ are projective equivalent if $N=N^{\prime}$ and if there is an automorphism of $\mathbb{P}^{N}$ mapping $X$ onto $X^{\prime}$. Theorem 3 will be proved in Theorems 4.1, 5.2, and 6.1. Here, to allow $X$ to be singular is important to classify varieties $X$ satisfying $\left(E_{m}\right)$ by induction on $m$ based on Lemma 3.2 .

As a consequence of Theorems 1-3, we have the following theorem.

Theorem 4. Let $X \subseteq \mathbb{P}^{N}$ be a nondegenerate smooth projective variety of dimension $n \geq 2$, codimension $e \geq 2$, and degree $d$. Suppose that $X$ is projectively equivalent to none of (1)-(3):

(1) A scroll $\mathbb{P}_{C}(\mathcal{F}) \subseteq \mathbb{P}^{N}$ for a locally free sheaf $\mathcal{F}$ on a smooth projective curve $C$.

(2) The Veronese surface $v_{2}\left(\mathbb{P}^{2}\right) \subseteq \mathbb{P}^{5}$.

(3) A birational-divisor of type $(\mu, 1)$ of a conical rational scroll for some $\mu \geq 2$.

Then the base locus of $\left|\mathcal{O}_{X}(d-n-e-1) \otimes \omega_{X}^{\vee}\right|$ is, at most, a finite set contained in $\mathcal{C}(X)$. Consequently, $\mathcal{O}_{X}(d-n-e-1) \otimes \omega_{X}^{\vee}$ is semiample, $\mathcal{O}_{X}(d-n-e) \otimes \omega_{X}^{\vee}$ is ample, and $\left|\mathcal{O}_{X}(d-n-e) \otimes \omega_{X}^{\vee}\right|$ is very ample on $X \backslash \mathcal{C}(X)$.

As applications, we have the following corollary, which will be proved in $\S 7$.

Corollary 5. Let $X \subseteq \mathbb{P}^{N}$ be a nondegenerate smooth projective variety of dimension $n \geq 2$, codimension e, and degree $d$. Then $\mathcal{O}_{X}$ is $(d-e)$-regular (i.e., $H^{i}\left(X, \mathcal{O}_{X}(d-e-i)\right)=0$ for all $\left.i>0\right)$ if $X$ is not projectively equivalent to any scroll over a smooth projective curve.

This result gives evidence of the regularity conjecture ([3] and [9, §4]), which asserts that a nondegenerate projective variety $X \subseteq \mathbb{P}^{N}$ of degree $d$ and codimension $e$ is $(d-e+1)$-regular (i.e., $H^{i}\left(\mathbb{P}^{N}, \mathcal{I}_{X} \otimes \mathcal{O}_{\mathbb{P}^{N}}(d-e+1)\right)=0$ for all $\left.i>1\right)$, since 
the assertion of the conjecture is equivalent to the two conditions: the surjectivity of $H^{0}\left(\mathbb{P}^{N}, \mathcal{O}_{\mathbb{P}^{N}}(d-e)\right) \rightarrow H^{0}\left(X, \mathcal{O}_{X}(d-e)\right)$ and the $(d-e)$-regularity of $\mathcal{O}_{X}$.

Also we will apply Theorem 4 to Property $\left(N_{p}\right)$ (Corollary 7.1 ) and the $\Delta$-genus and sectional genus of projective varieties (Corollaries 7.3, 7.6).

This paper is organized as follows. In $\S 1$, we recall generic inner projections and their extension morphisms. We also look at their relation in terms of exceptional sets of codimension one. In $\S 2$, we prove Theorem 1 . In $\S 3$, we prove properties of projective varieties satisfying $\left(E_{m}\right)$ which are useful in the proof of Theorem 3 . In $\S 4-6$, we classify projective varieties satisfying $\left(E_{1}\right),\left(E_{2}\right)$, and $\left(E_{m}\right)(e-1 \leq m \leq$ 3 ), respectively, and consequently prove Theorems 3 and 4 . In $\S 7$, we give some applications of Theorem 4 .

\section{Generic inner projections And their extensions}

In this section, let $X \subseteq \mathbb{P}^{N}$ be a nondegenerate (not necessarily smooth) projective variety of dimension $n \geq 1$ and codimension $e=N-n \geq 2$.

Notation 1.1 (Inner projection and extended projection). Suppose $n \geq 2$. Let $m$ be an integer with $1 \leq m \leq e-1$. Let $x_{1}, \ldots, x_{m}$ be general points of $X$ and let $\Lambda$ be the linear span $\left\langle x_{1}, \ldots, x_{m}\right\rangle$. Let $\pi_{\Lambda}: \mathbb{P}^{N} \backslash \Lambda \rightarrow \mathbb{P}^{N-m}$ be the linear projection from $\Lambda$, and let $\pi_{\Lambda, X}: X \backslash \Lambda \rightarrow \mathbb{P}^{N-m}$ be the restriction morphism of $\pi_{\Lambda}$. Let $\bar{X}_{\Lambda}$ be the closure of $\pi_{\Lambda}(X \backslash \Lambda)$. Let $\pi_{\Lambda, X}^{\prime}: X \backslash \Lambda \rightarrow \bar{X}_{\Lambda}$ be the induced morphism. We call it the induced projection. Since $\Lambda \cap X=\left\{x_{1}, \ldots, x_{m}\right\}$ as a scheme by Lemma 1.2 below, if we take the blowing up of $\sigma: \hat{X} \rightarrow X$ at $x_{1}, \ldots, x_{m}$, the rational map $\pi_{\Lambda, X}$ extends to the morphism $\hat{\pi}_{\Lambda, X}: \hat{X} \rightarrow \mathbb{P}^{N-m}$ such that $\hat{\pi}_{\Lambda, X}=\pi_{\Lambda, X} \circ \sigma$ as rational maps (see [15, (1.1)]). We call it the extended projection. In particular, when $m=1$, for the embedded tangent space $T_{x_{1}}(X) \subseteq \mathbb{P}^{N}$ to $X$ and for $\mathbb{E}_{x_{1}}:=\pi_{x_{1}}\left(T_{x_{1}}(X) \backslash\left\{x_{1}\right\}\right)$, we have

$$
\sigma^{-1}\left(X \cap T_{x_{1}}(X)\right)=\hat{\pi}_{x_{1}, X}^{-1}\left(\mathbb{E}_{x_{1}}\right),
$$

since $\hat{\pi}_{x_{1}, X}^{-1}\left(\mathbb{E}_{x_{1}}\right) \backslash \sigma^{-1}\left(x_{1}\right)=\sigma^{-1}\left(\pi_{x_{1}, X}^{-1}\left(\mathbb{E}_{x_{1}}\right)\right)=\sigma^{-1}\left(T_{x_{1}}(X) \cap X \backslash\left\{x_{1}\right\}\right)$. Hence

$$
X \backslash T_{x_{1}}(X) \rightarrow \bar{X}_{x_{1}} \backslash \mathbb{E}_{x_{1}}
$$

is a projective and quasi-finite morphism, and hence finite. Moreover, it is birational by generality of $x_{1} \in X$ and Lemma 1.2 below.

We recall the following well known lemma.

Lemma 1.2 (General position lemma). Let $m$ be an integer with $2 \leq m \leq e$. For general points $x_{1}, \ldots, x_{m} \in X \subseteq \mathbb{P}^{N}$ and its linear span $\left\langle x_{1}, \ldots, x_{m}\right\rangle \subseteq \mathbb{P}^{N}$, we have $l\left(X \cap\left\langle x_{1}, \ldots, x_{m}\right\rangle\right)=m$, namely $X \cap\left\langle x_{1}, \ldots, x_{m}\right\rangle=\left\{x_{1}, \ldots, x_{m}\right\}$ as a scheme.

Proof. First we prove the lemma for $m=2$ by induction on $n=\operatorname{dim} X$. For $n=1$, see for example [17, Lemma 1.1]. For $n \geq 2$, by contradiction, suppose a constructible subset

$$
\left\{\left(x_{1}, x_{2}\right) \mid x_{1} \neq x_{2} \text { and } l\left(X \cap\left\langle x_{1}, x_{2}\right\rangle\right) \geq 3\right\} \quad \subseteq \quad X \times X
$$

is dense, and hence contains an dense open subset $U$ of $X \times X$ (see [10, Ch. II, Ex 3.18] for constructible sets). For general $\left(x_{1}, x_{2}\right) \in U$, we may assume that $X \cap\left\langle x_{1}, x_{2}\right\rangle$ lies on the smooth locus $\operatorname{Sm} X$ of $X$, since for the projection $\pi_{x_{1}}: \mathbb{P}^{N} \backslash$ $\left\{x_{1}\right\} \rightarrow \mathbb{P}^{N-1}$ from a smooth point $x_{1}$ of $X, \operatorname{dim} \pi_{x_{1}}\left(X \backslash\left\{x_{1}\right\}\right)=\operatorname{dim} X$, whereas 
$\operatorname{dim} \pi_{x_{1}}(\operatorname{Sing} X) \leq n-1$ for the singular locus $\operatorname{Sing} X$ of $X$. By Bertini's Theorem, for a general hyperplane $H$ containing $\left\langle x_{1}, x_{2}\right\rangle$, the intersection $X^{n-1}:=X \cap H$ is irreducible and reduced (see [14, Lemma 3]). By the induction, the lemma holds for $X^{n-1}$. But $U \cap X^{n-1} \times X^{n-1}(\subseteq X \times X)$ contains $\left(x_{1}, x_{2}\right)$ and hence is a dense open subset of $X^{n-1} \times X^{n-1}$, a contradiction.

Now we prove the lemma for $3 \leq m \leq e$ by contradiction. Let $m$ be the smallest $m$ such that the lemma does not hold. A constructible subset

$\left\{\left(x_{1}, \ldots, x_{m}\right) \mid x_{1}, \ldots, x_{m}\right.$ are distinct and $\left.l\left(X \cap\left\langle x_{1}, \ldots, x_{m}\right\rangle\right) \geq m+1\right\} \subseteq X \times \cdots \times X$ is dense, and hence contains an dense open subset $U^{\prime}$. Let $\left(x_{1}, \ldots, x_{m}\right) \in U^{\prime}$ be a general element. By the minimality of $m, l\left(X \cap\left\langle x_{1}, \ldots, x_{m-1}\right\rangle\right)=m-1$. Hence, for the projection $\pi_{\Lambda}: \mathbb{P}^{N} \backslash \Lambda \rightarrow \mathbb{P}^{N-m+2}$ from $\Lambda:=\left\langle x_{1}, \ldots, x_{m-2}\right\rangle$, the closure $\bar{X}_{\Lambda}$ of $\pi_{\Lambda}(X \backslash \Lambda)$ is a nondegenerate projective variety of dimension $n$ in $\mathbb{P}^{N-m+2}$. Since $\bar{x}_{m-1}:=\pi_{\Lambda}\left(x_{m-1}\right)$ and $\bar{x}_{m}:=\pi_{\Lambda}\left(x_{m}\right)$ are general points of $\bar{X}_{\Lambda}$, we have for each $i=1, \ldots, m-2,\left\langle\bar{x}_{m-1}, \bar{x}_{m}\right\rangle \cap \pi_{\Lambda}\left(T_{x_{i}}(X) \backslash \Lambda\right)=\emptyset$, and for each $j=m-1$ and $m,\left\langle\bar{x}_{m-1}, \bar{x}_{m}\right\rangle \nsubseteq \pi_{\Lambda}\left(T_{x_{j}}(X)\right)$. Consequently $\left\langle x_{1}, \ldots, x_{m}\right\rangle \cap T_{x_{i}}(X)=x_{i}$ for each $i=1, \ldots, m$. Thus there exists $x_{0} \in X \cap\left\langle x_{1}, \ldots, x_{m}\right\rangle$ such that $x_{0} \neq x_{i}$ for all $i=1, \ldots, m$ and $x_{0} \notin \Lambda$. By the minimality of $m$, this means that the general secant line $\left\langle\bar{x}_{m-1}, \bar{x}_{m}\right\rangle$ meets with $\bar{X}_{\Lambda}$ in three distinct points. This contradicts the lemma for $m=2$.

By the next lemma, $\left(E_{m}\right)$ is equivalent to the condition for the extended projection $\hat{\pi}_{\Lambda, X}$ to have an exceptional set of codimension one.

Lemma 1.3. Suppose $n \geq 2$. Let $m$ be an integer with $1 \leq m \leq e-1$. Let $x_{1}, \ldots, x_{m} \in X$ be general points and let $\Lambda=\left\langle x_{1}, \ldots, x_{m}\right\rangle$ be the linear span. Keep the notation in Notation 1.1. Then

$$
\hat{E}_{x_{1}, \ldots, x_{m}}:=\left\{z \in \hat{X} \mid \operatorname{dim} \hat{\pi}_{\Lambda, X}^{-1}\left(\hat{\pi}_{\Lambda, X}(z)\right) \geq 1\right\}
$$

is a closed subset of $\hat{X}$, and we have

$$
\sigma\left(\hat{E}_{x_{1}, \ldots, x_{m}}\right) \supseteq E_{x_{1}, \ldots, x_{m}}(X) \supseteq E_{x_{1}, \ldots, x_{m}}^{0}(X)=\sigma\left(\hat{E}_{x_{1}, \ldots, x_{m}}\right) \backslash\left\{x_{1}, \ldots, x_{m}\right\} .
$$

Hence $\operatorname{dim} E_{x_{1}, \ldots, x_{m}}(X) \geq n-1$ if and only if $\operatorname{dim} \hat{E}_{x_{1}, \ldots, x_{m}} \geq n-1$.

Proof. By [18, I.6.3, Corollary], $\hat{E}_{x_{1}, \ldots, x_{m}}$ is closed. Hence $\sigma\left(\hat{E}_{x_{1}, \ldots, x_{m}}\right)$ is closed since $\sigma$ is projective. Let $E_{i}$ be the exceptional divisor on $\hat{X}$ over $x_{i}$. For $z \in \hat{X} \backslash$ $\bigcup_{i=1, \ldots, m} E_{i}, z \in \hat{E}_{x_{1}, \ldots, x_{m}}$ if and only if $\sigma(z) \in E_{x_{1}, \ldots, x_{m}}^{0}(X)$, since $\hat{\pi}_{\Lambda, X}\left(E_{i}\right) \cong E_{i}$ and $\hat{\pi}_{\Lambda, X}=\pi_{\Lambda, X} \circ \sigma$. This means $\sigma\left(\hat{E}_{x_{1}, \ldots, x_{m}}\right) \backslash\left\{x_{1}, \ldots, x_{m}\right\}=E_{x_{1}, \ldots, x_{m}}^{0}(X)$, and we have (1.3.1). For the second part, by (1.3.1), we have only to show that $E_{i} \nsubseteq \hat{E}_{x_{1}, \ldots, x_{m}}$ for all $i$. If $E_{i} \subseteq \hat{E}_{x_{1}, \ldots, x_{m}}$ for some $i$, from $\hat{\pi}_{\Lambda, X}\left(E_{i}\right) \cong E_{i}$ it follows that $\hat{\pi}_{\Lambda, X}^{-1}\left(\hat{\pi}_{\Lambda, X}\left(E_{i}\right)\right)=\hat{X}$ and $\hat{\pi}_{\Lambda, X}(\hat{X})=\hat{\pi}_{\Lambda, X}\left(E_{i}\right)$, which means $\hat{X} \subseteq \hat{E}_{x_{1}, \ldots, x_{m}}$, a contradiction.

\section{Proof of Theorem 1}

Before starting the proof of Theorem 1, recall that the base-point-freeness of $\left|\mathcal{O}_{X}(d-n-2) \otimes \omega_{X}^{\vee}\right|$ follows from observing that for a given point $x_{0} \in X$, the linear projection from a general $(e-2)$-dimensional linear subspace $\Lambda \subseteq \mathbb{P}^{N}$ induces a birational finite morphism from $X$ to a hypersurface in $\mathbb{P}^{n+1}$ such that it is isomorphic at $x_{0} \in X$ and its ramification divisor is a member of $\left|\mathcal{O}_{X}(d-n-2) \otimes \omega_{X}^{\vee}\right|$ 
off $x_{0}$ (see [2, (3.3)]). Theorem 1 will prove along this line. The key ingredients are the birational double-point formula (Lemma 2.1) and the isomorphic locus of a generic inner projection (Lemma 2.2).

We recall the birational double-point formula [12, II, 10.2.8]. Here we review its proof too, because it is not explicitly stated that the double-point divisor is supported on the nonisomorphic locus.

A divisor $E$ on a smooth projective variety $V$ is said to be $\varphi$-exceptional for a birational morphism $\varphi: V \rightarrow W$ if every irreducible component $Z$ of $E$ satisfies $\operatorname{dim} \varphi(Z) \leq \operatorname{dim} Z-1$. On the other hand, a surjective morphism $f: X \rightarrow Y$ of projective varieties is said to be isomorphic at $x \in X$ if $l\left(f^{-1}(f(x))\right)=1$ for the fibre $f^{-1}(f(x))=X \times_{Y} \operatorname{Spec} \mathbb{k}(f(x))$.

Lemma 2.1 (Birational double-point formula [12, II, 10.2.8]). Let $f: V \rightarrow M$ be $a$ morphism of smooth projective varieties of dimension $m$ and $m+1$ respectively. By $K_{V}$ and $K_{M}$ we denote their canonical divisors. Assume that $f$ induces a birational morphism $\varphi: V \rightarrow W$ onto a (singular) hypersurface $W \subseteq M$. Then there exist effective divisors $D$ and $E$ on $V$ such that $E$ is $\varphi$-exceptional and $f^{*}\left(K_{M}+W\right)-K_{V}$ is linearly equivalent to $D-E$. Moreover, if $\varphi$ is isomorphic at $x \in V$, then $x \notin \operatorname{Supp}(D-E)$.

Proof ([12, II, 10.2.8, p. 245]). By [12, II, 9.3.47 and 9.3.48, p. 179], there exists an ideal sheaf $\mathcal{J}$, i.e., the adjoint ideal $\mathcal{J}=\operatorname{adj}(W)$ of $W$, such that (1) $\mathcal{J}$ fits in an exact sequence $0 \rightarrow \mathcal{O}_{M}\left(K_{M}\right) \rightarrow \mathcal{O}_{M}\left(K_{M}+W\right) \otimes \mathcal{J} \rightarrow \varphi_{*} \mathcal{O}_{V}\left(K_{V}\right) \rightarrow 0$, and (2) $\mathcal{J}_{y}=\mathcal{O}_{M, y}$ at $y \in W$ by the inclusion if and only if $W$ is normal and has at worst rational singularities at $y$. Let $\mathfrak{a}$ be the restriction ideal sheaf $\mathcal{J} \mid W$ of $\mathcal{O}_{W}$. By restricting the sequence to $W$, we have $\left(\left.\mathcal{O}_{M}\left(K_{M}+W\right)\right|_{W}\right) \otimes \mathfrak{a} \cong$ $\varphi_{*} \mathcal{O}_{V}\left(K_{V}\right)$ since both are torsion-free $\mathcal{O}_{W}$-modules. By pulling this back to $V$, we have $\varphi^{*}\left(\left.\mathcal{O}_{M}\left(K_{M}+W\right)\right|_{W}\right) \otimes \varphi^{*} \mathfrak{a} \cong \varphi^{*} \varphi_{*} \mathcal{O}_{V}\left(K_{V}\right)$. The double dual $\left(\varphi^{*} \mathfrak{a}\right)^{\vee \vee}$ is an $\mathcal{O}_{V}$-ideal which is torsion-free of rank one, and from the inclusion $\left(\varphi^{*} \mathfrak{a}\right)^{\vee \vee} \subseteq \mathcal{O}_{V}$ we obtain $\left(\varphi^{*} \mathfrak{a}\right)^{\vee \vee}=\mathcal{O}_{V}(-D)$ for an effective divisor $D$ on $V$. Thus

$$
\varphi^{*}\left(\left.\mathcal{O}_{M}\left(K_{M}+W\right)\right|_{W}\right) \otimes \mathcal{O}_{V}(-D) \cong\left(\varphi^{*} \varphi_{*} \mathcal{O}_{V}\left(K_{V}\right)\right)^{\vee \vee} .
$$

On the other hand, since the natural map $\varphi^{*} \varphi_{*} \mathcal{O}_{V}\left(K_{V}\right) \rightarrow \mathcal{O}_{V}\left(K_{V}\right)$ factors through $\left(\varphi^{*} \varphi_{*} \mathcal{O}_{V}\left(K_{V}\right)\right)^{\vee \vee}$, we have $\left(\varphi^{*} \varphi_{*} \mathcal{O}_{V}\left(K_{V}\right)\right)^{\vee \vee} \cong \mathcal{O}_{V}\left(K_{V}-E\right)$ for some $\varphi$-exceptional divisor $E$, and $f^{*} \mathcal{O}_{M}\left(K_{M}+W\right) \otimes \mathcal{O}_{V}\left(-K_{V}\right)=\varphi^{*}\left(\left.\mathcal{O}_{M}\left(K_{M}+W\right)\right|_{W}\right) \otimes \mathcal{O}_{V}\left(-K_{V}\right) \cong$ $\mathcal{O}_{V}(D-E)$. For the second part, suppose $\varphi$ is isomorphic at $x \in V$. Clearly $x \notin E$. On the other hand, $W$ is smooth at $\varphi(x)$, and from (2) above, it follows that $\mathfrak{a}_{\varphi(x)}=\mathcal{O}_{W, \varphi(x)}$ by the inclusion. Hence the inclusion $\left(\varphi^{*} \mathfrak{a}\right)_{x}^{\vee \vee} \subseteq \mathcal{O}_{V, x}$ is surjective and thus $x \notin D$.

Lemma 2.2. Let $X \subseteq \mathbb{P}^{N}$ be a nondegenerate (not necessarily smooth) projective variety of codimension $e \geq 2$. Let $m$ be a positive integer with $1 \leq m \leq e-1$. Let $x$ be a point of $\operatorname{Sm} X \backslash \mathcal{C}(X)$. Then for general points $x_{1}, \ldots, x_{m}$ of $X$ and $\Lambda:=\left\langle x_{1}, \ldots, x_{m}\right\rangle$, the induced projection $\pi_{\Lambda, X}^{\prime}: X \backslash \Lambda \rightarrow \bar{X}_{\Lambda}$ in Notation 1.1 is isomorphic at $x$.

Proof. We have only to show the following.

Claim. For general $x_{1} \in X$, the induced projection $\pi_{x_{1}, X}^{\prime}: X \backslash\left\{x_{1}\right\} \rightarrow \bar{X}_{x_{1}}$ is isomorphic at $x$, and moreover if $e \geq 3$, then $\bar{x}:=\pi_{x_{1}}(x) \notin \mathcal{C}\left(\bar{X}_{x_{1}}\right)$. 
(If the above Claim is obtained, the lemma follows from the claim by the induction on $m$, by taking the projection from a general point of the image of $X$.) By the generality of $x_{1}$ and by $x \notin \mathcal{C}(X)$, we may assume that $x_{1}$ is a smooth point of $X$ and $l\left(X \cap\left\langle x, x_{1}\right\rangle\right)=2$, and consequently $x_{1} \notin T_{x}(X)$ and $x \notin T_{x_{1}}(X)$. Let $\sigma: \hat{X} \rightarrow X$ be the blowing up of $X$ at $x_{1}$ and let $\hat{\pi}_{x_{1}, X}: \hat{X} \rightarrow \mathbb{P}^{N-1}$ be the extended projection of $\pi_{x_{1}, X}: X \backslash\left\{x_{1}\right\} \rightarrow \mathbb{P}^{N-1}$. Set $\mathbb{E}_{x_{1}}:=\pi_{x_{1}}\left(T_{x_{1}}(X) \backslash\left\{x_{1}\right\}\right)$. By (1.1.2), the induced morphism

$$
X \backslash T_{x_{1}}(X)\left(\cong \hat{X} \backslash \sigma^{-1}\left(T_{x_{1}}(X) \cap X\right)\right) \rightarrow \bar{X}_{x_{1}} \backslash \mathbb{E}_{x_{1}}
$$

from $\hat{\pi}_{x_{1}, X}$ is a finite morphism. Since $l\left(X \cap\left\langle x, x_{1}\right\rangle\right)=2, \pi_{x_{1}, X}^{\prime}$ is isomorphic at $x$, and hence $\bar{X}_{x_{1}}$ is smooth at $\bar{x}:=\pi_{x_{1}}(x)$ with $T_{\bar{x}}\left(\bar{X}_{x_{1}}\right)=\pi_{x_{1}}\left(T_{x}(X)\right)$.

Next we will show that $\bar{x}=\pi_{x_{1}}(x) \notin \mathcal{C}\left(\bar{X}_{x_{1}}\right)$ if $e \geq 3$. By contradiction, we suppose that $\bar{x} \in \mathcal{C}\left(\bar{X}_{x_{1}}\right)$, and hence for general $y \in X$ and the image $\bar{y}=\pi_{x_{1}}(y)$,

$$
l\left(\bar{X}_{x_{1}} \cap\langle\bar{x}, \bar{y}\rangle\right) \geq 3 .
$$

By the generality of $y \in X$, we may assume that $l(X \cap\langle x, y\rangle)=2$ and $\pi_{x_{1}, X}^{\prime}$ is isomorphic at $y$ with $T_{\bar{y}}\left(\bar{X}_{x_{1}}\right)=\pi_{x_{1}}\left(T_{y}(X)\right)$. Moreover, $y \notin\left\langle T_{x}(X), x_{1}\right\rangle$ and $y \notin\left\langle T_{x_{1}}(X), x\right\rangle$, which imply $\bar{y} \notin T_{\bar{x}}\left(\bar{X}_{x_{1}}\right)$ and $\langle\bar{x}, \bar{y}\rangle \cap \mathbb{E}_{x_{1}}=\emptyset$, respectively. By $\bar{x} \in \operatorname{Sm} \bar{X}_{x_{1}}$ and the generality of $y$, we have $\bar{x} \notin T_{\bar{y}}\left(\bar{X}_{x_{1}}\right)$. These together with (2.2.1) imply that there exists a point $z \in X \backslash\{x, y\}$ whose image $\bar{z}:=\pi_{x_{1}}(z)$ lies on $\bar{X}_{x_{1}} \cap\langle\bar{x}, \bar{y}\rangle$. Hence $z \in\left\langle x_{1}, x, y\right\rangle \backslash\left(\left\langle x_{1}, x\right\rangle \cup\langle x, y\rangle\right)$ by $l\left(X \cap\left\langle x, x_{1}\right\rangle\right)=2$ and $l(X \cap\langle x, y\rangle)=2$. Consider the closure $\bar{X}_{x} \subseteq \mathbb{P}^{N-1}$ of the image of $X$ by the projection from $x$. The line connecting the images of $x_{1}$ and $y$ in $\bar{X}_{x}$ is a general secant line to $\bar{X}_{x}$, but it is a trisecant line. This contradicts Lemma 1.2 since $\operatorname{codim}\left(\bar{X}_{x}, \mathbb{P}^{N-1}\right)=e-1 \geq 2$.

Proof of Theorem 1. Let $x$ be a point of $X \backslash \mathcal{C}(X)$. Choose general points $x_{1}, \ldots, x_{m}$ $\in X$ and consider the linear projection $\pi_{\Lambda}: \mathbb{P}^{N} \backslash \Lambda \rightarrow \mathbb{P}^{N-m}$ from the linear span $\Lambda:=\left\langle x_{1}, \ldots, x_{m}\right\rangle$. Let $\pi_{\Lambda, X}: X \backslash \Lambda \rightarrow \mathbb{P}^{N-m}$ be the restriction morphism and let $\bar{X}_{\Lambda}$ be the closure of $\pi_{\Lambda}(X \backslash \Lambda)$ in $\mathbb{P}^{N-m}$. Because of the generality of $x_{1}, \ldots, x_{m} \in X$, we have

(1) $\Lambda \cap X=\left\{x_{1}, \ldots, x_{m}\right\}$ as a scheme by Lemma 1.2 ,

(2) $\operatorname{dim} E_{x_{1}, \ldots, x_{m}}(X) \leq n-2$ by the assumption on $\left(E_{m}\right)$, and

(3) the induced projection $\pi_{\Lambda, X}^{\prime}: X \backslash \Lambda \rightarrow \bar{X}_{\Lambda}$ is isomorphic at $x$ by Lemma 2.2 .

Let $\sigma: \hat{X} \rightarrow X$ be the blowing up of $X$ at $x_{1}, \ldots, x_{m}$ and let $E_{i}$ be the exceptional divisor over $x_{i}$. The extended projection $\hat{\pi}_{\Lambda, X}: \hat{X} \rightarrow \mathbb{P}^{N-m}$ (see Notation 1.1) has no exceptional divisor by (2) and Lemma 1.3. Set $\bar{x}:=\pi_{\Lambda, X}(x)$. Let $\Lambda^{\prime}$ be a general $(N-m-n-2)$-plane in $\mathbb{P}^{N-m}$ such that $\Lambda^{\prime} \cap \bar{X}_{\Lambda}=\emptyset$ and $\Lambda^{\prime} \cap\left(T_{\bar{x}}\left(\bar{X}_{\Lambda}\right) \cup\right.$ Cone $\left.\left(\bar{x}, \bar{X}_{\Lambda}\right)\right)=\emptyset$ and consider the projection $\pi_{\Lambda^{\prime}}: \mathbb{P}^{N-m} \backslash \Lambda^{\prime} \rightarrow \mathbb{P}^{n+1}$. Let $\overline{\bar{X}}$ be the image $\pi_{\Lambda^{\prime}}\left(\bar{X}_{\Lambda}\right)$. Consequently the induced projection $\pi_{\Lambda^{\prime}, \bar{X}_{\Lambda}}^{\prime}: \bar{X}_{\Lambda} \rightarrow \overline{\bar{X}}$ is a finite morphism, which is isomorphic at $\bar{x}$. Let

$$
\pi: X \backslash \Lambda \rightarrow \overline{\bar{X}} \quad \text { and } \hat{\pi}: \hat{X} \rightarrow \overline{\bar{X}}
$$

be the induced morphisms from $\pi_{\Lambda^{\prime}} \circ \pi_{\Lambda, X}$ and $\pi_{\Lambda^{\prime}} \circ \hat{\pi}_{\Lambda, X}$ respectively. Hence there is no $\hat{\pi}$-exceptional divisor, and $\hat{\pi}$ is isomorphic at $\sigma^{-1}(x)$. By Lemma 2.1, there exists an effective divisor $\mathbb{D}(\hat{\pi}) \in\left|\hat{\pi}^{*} \omega_{\bar{X}} \otimes \omega_{\hat{X}}^{\vee}\right|$ such that $\sigma^{-1}(x) \notin \mathbb{D}(\hat{\pi})$ for the dualizing line bundle $\omega_{\overline{\bar{X}}}$ of $\overline{\bar{X}}$. By the smoothness of $X$ at $x_{1}, \ldots, x_{m}$, the divisor $\mathbb{D}(\pi)$ of the closure of $\sigma\left(\left.\mathbb{D}(\hat{\pi})\right|_{\hat{X} \backslash E_{1} \cup \ldots \cup E_{m}}\right)$ is an effective Cartier divisor 
such that $x \notin \mathbb{D}(\pi)$. On the other hand, $\pi^{*} \omega_{\bar{X}} \otimes \omega_{X}^{\vee} \otimes \mathcal{O}_{X}(-\mathbb{D}(\pi)) \cong \mathcal{O}_{X}$ on $X \backslash\left\{x_{1}, \ldots, x_{m}\right\}$, since $\hat{\pi}^{*} \omega_{\bar{X}} \otimes \omega_{\hat{X}}^{\vee} \otimes \mathcal{O}_{\hat{X}}(-\mathbb{D}(\hat{\pi})) \cong \mathcal{O}_{\hat{X}}$ on $\hat{X}$. Noting that $\pi^{*} \omega_{\bar{X}}$ extends to $\mathcal{O}_{X}(d-m-n-2)$ because of $n \geq 2$ and the smoothness of $X$, we have $\mathbb{D}(\pi) \in\left|\mathcal{O}_{X}(d-m-n-2) \otimes \omega_{X}^{\vee}\right|$ with $x \notin \mathbb{D}(\pi)$. Now considering the divisors $\mathbb{D}(\pi)$ for all $x \notin \mathcal{C}(X)$, we have $\operatorname{Bs}\left|\mathcal{O}_{X}(d-m-n-2) \otimes \omega_{X}^{\vee}\right| \subseteq \mathcal{C}(X)$, and hence $\left|\mathcal{O}_{X}(d-m-n-1) \otimes \omega_{X}^{\vee}\right|$ is very ample on $X \backslash \mathcal{C}(X)$, which is the first part. If $\operatorname{dim} \mathcal{C}(X) \leq 0$, by Zariski-Fujita's Theorem ([5], [19], see also [12, I, 2.1.32, p. 132]) $\mathcal{O}_{X}(d-m-n-2) \otimes \omega_{X}^{\vee}$ is semiample. Hence $\mathcal{O}_{X}(d-m-n-1) \otimes \omega_{X}^{\vee}$ is ample.

\section{Some Properties of $\left(E_{m}\right)$}

In this section, let $X \subseteq \mathbb{P}^{N}$ be a nondegenerate projective variety of dimension $n \geq 2$, degree $d$ and codimension $e \geq 2$. We will prove useful lemmas in $\S 4-6$.

Lemma 3.1. Let $m$ be an integer with $1 \leq m \leq e-1$. Suppose $X$ satisfies $\left(E_{m}\right)$ but not $\left(E_{m-1}\right)$. Then for general points $x_{1}, \ldots, x_{m} \in X$, every $(n-1)$-dimensional subvariety $D$ of $E_{x_{1}, \ldots, x_{m}}(X)$ passes through $x_{1}, \ldots, x_{m}$.

Proof. Let $\Lambda$ be the linear span of $x_{1}, \ldots, x_{m}$ in $\mathbb{P}^{N}$. By Lemma 1.2, we have

$$
D \cap \Lambda \subseteq X \cap \Lambda=\left\{x_{1}, \ldots, x_{m}\right\}
$$

as schemes. By contradiction, suppose $x_{i_{0}} \notin D$ for some $1 \leq i_{0} \leq m$. Let $\Lambda^{\prime}$ be the $(m-2)$-dimensional linear subspace spanned by $\left\{x_{1}, \ldots, x_{m}\right\} \backslash\left\{x_{i_{0}}\right\}$. For the linear projection $\pi_{\Lambda^{\prime}}: \mathbb{P}^{N} \rightarrow \mathbb{P}^{N-m+1}$ from $\Lambda^{\prime}$, we have $\operatorname{dim} \pi_{\Lambda^{\prime}}\left(D \backslash \Lambda^{\prime}\right)=n-1$ since $X$ does not satisfy $\left(E_{m-1}\right)$. Let $\bar{D}_{\Lambda^{\prime}}$ be the closure of $\pi_{\Lambda^{\prime}}\left(D \backslash \Lambda^{\prime}\right)$. Note that $\bar{D}_{\Lambda^{\prime}}=\pi_{\Lambda^{\prime}}\left(D \backslash \Lambda^{\prime}\right) \cup \bigcup_{x_{i} \in \Lambda^{\prime} \cap D} \pi_{\Lambda^{\prime}}\left(T C_{x_{i}}(D) \backslash \Lambda^{\prime}\right)$ for the tangent cone $T C_{x_{i}}(D)$ to $D$ at $x_{i}$. Then $\bar{D}_{\Lambda^{\prime}}$ does not contain the image $\bar{x}_{i_{0}}:=\pi_{\Lambda^{\prime}}\left(x_{i_{0}}\right)$. Indeed, if $\bar{x}_{i_{0}} \in \bar{D}_{\Lambda^{\prime}}$, then $x_{i_{0}} \in\left\langle\Lambda^{\prime}, z\right\rangle$ for some $z \in D \backslash \Lambda^{\prime}$ or $x_{i_{0}} \in\left\langle T C_{x_{i}}(D), \Lambda^{\prime}\right\rangle \subseteq\left\langle T_{x_{i}}(X), \Lambda^{\prime}\right\rangle$ for some $i \neq i_{0}$, both of which contradict (3.1.1). Hence, for the linear projection $\pi_{\bar{x}_{i_{0}}}: \mathbb{P}^{N-m+1} \rightarrow \mathbb{P}^{N-m}$ from $\bar{x}_{i_{0}}$, we have $\operatorname{dim} \pi_{\bar{x}_{i_{0}}}\left(\bar{D}_{\Lambda^{\prime}}\right)=n-1$. But this contradicts $\operatorname{dim} \pi_{\Lambda}(D \backslash \Lambda)<n-1$ by $\left(E_{m}\right)$. Consequently $D \cap \Lambda=X \cap \Lambda$.

Lemma 3.2. Suppose $e \geq 3$. Let $m$ be an integer with $2 \leq m \leq e-1$. Suppose that $X$ satisfies $\left(E_{m}\right)$ but not $\left(E_{m-1}\right)$. Then for general $x \in X$ the closure $\bar{X}_{x}$ of image $\pi_{x}(X \backslash\{x\})$ by the projection $\pi_{x}$ from $x$ satisfies $\left(E_{m-1}\right)$ but not $\left(E_{m-2}\right)$.

Proof. First we will prove that $\bar{X}_{x}$ satisfies $\left(E_{m-1}\right)$. Let $\bar{x}_{2}, \ldots, \bar{x}_{m} \in \bar{X}_{x}$ be general points and let $x_{2}, \ldots, x_{m}$ be the corresponding general points of $X$ with $\pi_{x}\left(x_{i}\right)=\bar{x}_{i}$. By the assumption on $\left(E_{m}\right)$, there exists an $(n-1)$-dimensional subvariety $D$ of $E_{x, x_{2}, \ldots, x_{m}}(X)$. The closure $\bar{D}_{x}\left(\subseteq \bar{X}_{x}\right)$ of $\pi_{x}(D \backslash\{x\})$ is a subvariety of $E_{\bar{x}_{2}, \ldots, \bar{x}_{m}}\left(\bar{X}_{x}\right)$, and $\operatorname{dim} \bar{D}_{x}=n-1$ by the assumption on $\left(E_{m-1}\right)$ for $X$. Therefore $\bar{X}_{x}$ satisfies $\left(E_{m-1}\right)$. Next we will prove that $\bar{X}_{x}$ does not satisfy $\left(E_{m-2}\right)$ for $m \geq 3$. By contradiction, suppose that $\bar{X}_{x}$ satisfies $\left(E_{m-2}\right)$. For general points $\bar{x}_{2}, \ldots, \bar{x}_{m-1} \in \bar{X}_{x}$ and the corresponding points $x_{2}, \ldots, x_{m-1}$ of $X$, we have $\operatorname{dim} E_{\bar{x}_{2}, \ldots, \bar{x}_{m-1}}\left(\bar{X}_{x}\right)=n-1$. But $\operatorname{dim} E_{x, x_{2}, \ldots, x_{m-1}}(X)<n-1$ by the assumption on $\left(E_{m-1}\right)$ for $X$. By the generality of $\bar{x}_{2}, \ldots, \bar{x}_{m-1}(m \leq e-1)$ in $\bar{X}_{x}, E_{\bar{x}_{2}, \ldots, \bar{x}_{m-1}}\left(\bar{X}_{x}\right)$ does not contain $\mathbb{E}_{x}:=\pi_{x}\left(T_{x}(X) \backslash\{x\}\right)$. Hence, for an $(n-1)$-dimensional subvariety $B^{\prime}$ of $E_{\bar{x}_{2}, \ldots, \bar{x}_{m-1}}\left(\bar{X}_{x}\right)$, we have an $(n-1)$ dimensional subvariety $B$ of $X$ such that $B^{\prime}$ is the closure of $\pi_{x}(B \backslash x)$. Consequently, $\operatorname{dim} \pi_{\left\langle x, x_{2}, \ldots, x_{m-1}\right\rangle}(B) \leq n-2$ and $B \subseteq E_{x, x_{2}, \ldots, x_{m-1}}(X)$, which contradicts $\operatorname{dim} E_{x, x_{2}, \ldots, x_{m-1}}(X)<n-1$. 
Remark 3.3. In Lemma 3.2, we have proved that if $D$ is an $(n-1)$-dimensional subvariety of $X$ with $D \subseteq E_{x, x_{2}, \ldots, x_{m}}(X)$, then the closure $\bar{D}_{x}$ of $\pi_{x}(D \backslash\{x\})$ is an $(n-1)$-dimensional subvariety of $\bar{X}_{x}$ with $\bar{D}_{x} \subseteq E_{\bar{x}_{2}, \ldots, \bar{x}_{m}}\left(\bar{X}_{x}\right)$.

For $X \subseteq \mathbb{P}^{N}$ satisfying $\left(E_{m}\right)$ and for general points $x_{1}, \ldots, x_{m} \in X(1 \leq m \leq$ $e-1)$, by $D_{x_{1}, \ldots, x_{m}}^{0}(X)$ we denote the union of the $(n-1)$-dimensional irreducible components of $E_{x_{1}, \ldots, x_{m}}^{0}(X)$, and by $D_{x_{1}, \ldots, x_{m}}(X)$ the closure of $D_{x_{1}, \ldots, x_{m}}^{0}(X)$ with reduced induced structure. Hence $D_{x_{1}, \ldots, x_{m}}(X)$ is the union of all $(n-1)$ dimensional components of $E_{x_{1}, \ldots, x_{m}}(X)$ (see Lemma 1.3). We will construct a family of $D_{x_{1}, \ldots, x_{m}}(X)$.

Lemma 3.4. Let $m$ be an integer with $1 \leq m \leq e-1$. Suppose that $X$ satisfies $\left(E_{m}\right)$. Then the subset

$$
\left\{\left(x_{1}, \ldots, x_{m}\right) \mid \begin{array}{l}
\operatorname{dim}\left\langle x_{1}, \ldots, x_{m}\right\rangle=m-1 \text { and } \\
\operatorname{dim} E_{x_{1}, \ldots, x_{m}}(X) \geq n-1
\end{array}\right\} \subseteq X \times \cdots \times X
$$

contains a dense open subset $\mathfrak{X}_{m}$ of $X \times \cdots \times X$. Moreover, there exists a closed subscheme $\mathcal{D}$ of $\mathfrak{X}_{m} \times X$ projective over $\mathfrak{X}_{m}$ such that the first projection $p_{\mathcal{D}}: \mathcal{D} \rightarrow$ $\mathfrak{X}_{m}$ is flat over $\mathfrak{X}_{m}$ and each fibre of $p_{\mathcal{D}}$ over $\left(x_{1}, \ldots, x_{m}\right) \in \mathfrak{X}_{m}$ is reduced and equal to $D_{x_{1}, \ldots, x_{m}}(X)$.

Proof. For $l=m+1$ and $m$, by $I(l)$ we denote the dense open subset

$$
\left\{\left(x_{1}, \ldots, x_{l}\right) \mid x_{1}, \ldots, x_{l} \in X \text { are distinct and } \operatorname{dim}\left\langle x_{1}, \ldots, x_{l}\right\rangle=l-1\right\}
$$

$$
\subseteq X \times \cdots \times X
$$

The subset

$$
I(m+1)_{1}:=\left\{\left(x_{1}, \ldots, x_{m}, x\right) \in I(m+1) \mid \operatorname{dim} X \cap\left\langle x_{1}, \ldots, x_{m}, x\right\rangle \geq 1\right\}
$$

is closed in $I(m+1)$. Indeed, for the closed subset $W:=\left\{\left(x_{1}, \ldots, x_{m}, x, z\right) \in\right.$ $\left.I(m+1) \times X \mid z \in\left\langle x_{1}, \ldots, x_{m}, x\right\rangle\right\}$ of $I(m+1) \times X$ and for the projection $p_{1}$ : $W \rightarrow I(m+1)$ which is projective and surjective, $I(m+1)_{1}$ is the set of all $\left(x_{1}, \ldots, x_{m}, x\right) \in I(m+1)$ with $\operatorname{dim} p_{1}^{-1}\left(x_{1}, \ldots, x_{m}, x\right) \geq 1$, and hence it is closed (see [18, I.6.3, Corollary]. Moreover $I(m+1)_{1}$ is dominating $I(m)$ by the first projection $p: I(m+1)_{1} \rightarrow I(m)$ because of the assumption $\left(E_{m}\right)$. Let $\left\{Z_{i}\right\}$ be the irreducible components of $I(m+1)_{1}$ dominating $I(m)$ and let $p_{Z_{i}}: Z_{i} \rightarrow I(m)$ be the first projection. Since the image of a constructible set is a constructible subset (see [10, II.Ex 3.18 and 3.19]), $\bigcap_{i} p\left(Z_{i}\right)$ contains a dense open subset $\mathfrak{X}_{m}$ of $X \times \cdots \times X$ such that $\mathfrak{X}_{m}$ is disjoint from the images of other irreducible components of $I(m+1)_{1}$ not dominating $I(m)$ and $\operatorname{dim} p_{Z_{i}}^{-1}\left(x_{1}, \ldots, x_{m}\right)=\operatorname{dim} Z_{i}-\operatorname{dim} I(m)$ for all $\left(x_{1}, \ldots, x_{m}\right) \in \mathfrak{X}_{m}$ (see [18, I.6.3, Theorem 7]). Replacing $\mathfrak{X}_{m}$, by Lemma 1.2, we may assume that $l\left(X \cap\left\langle x_{1}, \ldots, x_{m}\right\rangle\right)=m$ for each $\left(x_{1}, \ldots, x_{m}\right) \in \mathfrak{X}_{m}$. For each $Z_{i}$ with $\operatorname{dim} Z_{i}=\operatorname{dim} I(m+1)_{1}$, the subset of all $\left(x_{1}, \ldots, x_{m}\right) \in \mathfrak{X}_{m}$ with $\operatorname{dim} p_{Z_{i}}^{-1}\left(x_{1}, \ldots, x_{m}\right) \geq n-1$ is closed in $\mathfrak{X}_{m}$ and dense by the assumption $\left(E_{m}\right)$, and hence is equal to $\mathfrak{X}_{m}$. Let $\mathcal{E}^{0}$ be the union of $Z_{i} \cap p^{-1}\left(\mathfrak{X}_{m}\right)$ and let $\mathcal{D}^{0}$ be the union of $Z_{i} \cap p^{-1}\left(\mathfrak{X}_{m}\right)$ with $\operatorname{dim} Z_{i}=\operatorname{dim} I(m+1)_{1}$, both of which have the reduced induced structure. By construction, for the first projections $p_{\mathcal{E}^{0}}: \mathcal{E}^{0} \rightarrow \mathfrak{X}_{m}$ and $p_{\mathcal{D}^{0}}: \mathcal{D}^{0} \rightarrow \mathfrak{X}_{m}$, we have $p_{\mathcal{E}^{0}}^{-1}\left(x_{1}, \ldots, x_{m}\right)=E_{x_{1}, \ldots, x_{m}}^{0}(X)$ and $p_{\mathcal{D}^{0}}^{-1}\left(x_{1}, \ldots, x_{m}\right)=$ $D_{x_{1}, \ldots, x_{m}}^{0}(X)$ as sets. Let $\mathcal{D}$ be the closure of $\mathcal{D}^{0}$ in $\mathfrak{X}_{m} \times X$ with the reduced induced structure and let $p_{\mathcal{D}}: \mathcal{D} \rightarrow \mathfrak{X}_{m}$ be the first projection. Replacing $\mathfrak{X}_{1}$ with a dense open subset and $\mathcal{D}$ with the pull-back, we may assume that $p_{\mathcal{D}}$ is flat by generic 
flatness (see [13, Lecture 8, Proposition]), and hence each fibre of $p_{\mathcal{D}}$ is pure $(n-1)$ dimensional (see [10, III.9.6]). Replacing $\mathfrak{X}_{m}$ and $\mathcal{D}$ again, we may assume that $\operatorname{dim} p_{\mathcal{D}}^{-1}\left(x_{1}, \ldots, x_{m}\right) \backslash p_{\mathcal{D}^{0}}^{-1}\left(x_{1}, \ldots, x_{m}\right)<n-1$ for all $\left(x_{1}, \ldots, x_{m}\right) \in \mathfrak{X}_{m}$. Hence, as sets, $p_{\mathcal{D}}^{-1}\left(x_{1}, \ldots, x_{m}\right)=D_{x_{1}, \ldots, x_{m}}(X)$, the closure of $p_{\mathcal{D}^{0}}^{-1}\left(x_{1}, \ldots, x_{m}\right)$ in $X$. By the generic smoothness and generic property of Serre's condition $\left(\mathrm{S}_{1}\right)([4,3.3 .10])$, general fibres of $p_{\mathcal{D}}$ are reduced (see [4, 3.3.13]). By replacing $\mathfrak{X}_{m}$ and $\mathcal{D}$ again, we have the required property.

\section{Projective varieties Satisfying $\left(E_{1}\right)$}

Theorem 4.1. Let $X \subseteq \mathbb{P}^{N}$ be a nondegenerate projective variety of dimension $n \geq 2$ and codimension $e$. Suppose that $e \geq 2$ and $X$ satisfies $\left(E_{1}\right)$. Then for a general point $x \in X, D_{x}(X)(\subseteq X)$ in $\S 3$ is an $(n-1)$-dimensional linear subspace of $\mathbb{P}^{N}$ through $x$. Consequently, $X$ is projectively equivalent to a scroll over a curve. If $X$ is furthermore smooth, then $X$ is projectively equivalent to a scroll $\mathbb{P}_{C}(\mathcal{F}) \subseteq \mathbb{P}^{N}$ for a locally free sheaf $\mathcal{F}$ of rank $n$ on a smooth projective curve $C$.

Proof. By the generality of $x$, we may assume that $x \in \operatorname{Sm} X$ and $x \in \mathfrak{X}_{1}$ in Lemma 3.4. First we prove that $D_{x}(X)$ is a reduced (possibly reducible) hypersurface in $T_{x}(X)$ which is a cone with vertex $x$. We have only to prove that each irreducible component $D$ of $D_{x}(X)$ has the same property. Consider the linear projection $\pi_{x}: \mathbb{P}^{N} \backslash\{x\} \rightarrow \mathbb{P}^{N-1}$ from $x$. Let $\bar{X}_{x}$ (resp. $\bar{D}_{x}$ ) be the closure of $\pi_{x}(X \backslash\{x\})$ (resp. $\left.\pi_{x}(D \backslash\{x\})\right)$. By assumption on $\left(E_{1}\right), D$ is the cone over $\bar{D}_{x}$ with vertex $x$ and $\operatorname{dim} \bar{D}_{x}=n-2$, and hence $D$ is a hypersurface in the embedded tangent space $T_{x}(X)$ to $X$ at $x$.

Next we will prove $\operatorname{deg} D_{x}(X)=1$ for $x \in \mathfrak{X}_{1}$ by induction on $n$. When $n=2$, if $\operatorname{deg} D_{x}(X) \geq 2$, then, by the first part, $D_{x}(X)$ contains at least two lines as irreducible components, which is impossible by Proposition 4.2 below. When $n \geq 3$, we will prove the following.

Claim. Suppose $n \geq 3$. If a hyperplane $H \subseteq \mathbb{P}^{N}$ is general and if $x \in \mathfrak{X}_{1} \cap$ $H(\subseteq X \cap H)$ is general, then for any smooth point $z \in \operatorname{Sm} D_{x}(X)$, we have $H \cap$ $T_{x}(X) \nsupseteq T_{z}\left(D_{x}(X)\right)$. Moreover, if Sing $D_{x}(X) \supsetneq\{x\}$, then $\operatorname{dim} H \cap \operatorname{Sing} D_{x}(X)<$ $\operatorname{dim} \operatorname{Sing} D_{x}(X)$.

In fact, the claim implies that for a general hyperplane $H \subseteq \mathbb{P}^{N}$ and for a general point $x \in \mathfrak{X}_{1} \cap H, \operatorname{Sm}\left(D_{x}(X) \cap H\right) \supseteq\left(\operatorname{Sm} D_{x}(X)\right) \cap H$ and $D_{x}(X) \cap H$ satisfies Serre's condition $\left(R_{0}\right)$. Hence $D_{x}(X) \cap H$ is reduced and $\operatorname{deg} D_{x}(X) \cap H=\operatorname{deg} D_{x}(X)(\geq 1)$, since $D_{x}(X) \cap H$ is a hypersurface in $T_{x}(X) \cap H$. Moreover, $D_{x}(X) \cap H$ is a subset of $E_{x}(X \cap H)$ and $X \cap H$ satisfies $\left(E_{1}\right)$. Note $X \cap H$ is a projective variety by Bertini's Theorem. By induction, $\operatorname{deg} D_{x}(X)=\operatorname{deg} D_{x}(X) \cap H \leq 1$ for general $x \in \mathfrak{X}_{1} \cap H$. Hence $\operatorname{deg} D_{x}(X)=1$ for general $x \in \mathfrak{X}_{1}$ by the flatness of $p_{\mathcal{D}}: \mathcal{D} \rightarrow \mathfrak{X}_{1}$ in Lemma 3.4.

Now we will prove the Claim. For $p_{\mathcal{D}}: \mathcal{D} \rightarrow \mathfrak{X}_{1}$ in Lemma 3.4, we may assume $\mathfrak{X}_{1} \subseteq \operatorname{Sm} X$. By $\mathcal{D}_{x}$ we denote fibre $p_{\mathcal{D}}^{-1}(x)=D_{x}(X)$ over $x \in \mathfrak{X}_{1}$. Consider the subset $U$ of $\left(\mathbb{P}^{N}\right)^{\vee} \times \mathfrak{X}_{1}$,

$$
U:=\left\{(H, x) \mid x \in H, T_{x}(X) \not \subset H, \text { and } \begin{array}{l}
(a) \quad T_{y}\left(\mathcal{D}_{x}\right) \subseteq H \text { for some } y \in \operatorname{Sm} \mathcal{D}_{x}, \quad \text { or } \\
\text { (b) } \operatorname{Sing} \mathcal{D}_{x} \supsetneq\{x\} \\
\text { and } \operatorname{dim}\left(\operatorname{Sing} \mathcal{D}_{x}\right) \cap H=\operatorname{dim} \operatorname{Sing} \mathcal{D}_{x}
\end{array}\right\},
$$


which is an algebraic family. Here $\left(\mathbb{P}^{N}\right)^{\vee}$ denotes the dual projective space. Fix a point $x_{0} \in \mathfrak{X}_{1}$. Since $\mathcal{D}_{x_{0}}=D_{x_{0}}(X)$ is a cone with vertex $x_{0}$, the set of hyperplanes $H$ through $x_{0}$ containing $T_{y}\left(\mathcal{D}_{x_{0}}\right)$ for some $y \in \operatorname{Sm} \mathcal{D}_{x_{0}}$ has the dimension at most $n-2+e$. On the other hand, when $\left\{x_{0}\right\} \subsetneq \operatorname{Sing} \mathcal{D}_{x_{0}}$, the set of hyperplanes $H$ through $x_{0}$ with $\operatorname{dim}\left(\operatorname{Sing} \mathcal{D}_{x_{0}}\right) \cap H=\operatorname{dim} \operatorname{Sing} \mathcal{D}_{x_{0}}$ has dimension at most $n-2+e$, since $\operatorname{dim} \operatorname{Sing} \mathcal{D}_{x_{0}} \geq 1$. Consequently, the fibre $U_{x_{0}}$ of $U \rightarrow \mathfrak{X}_{1}$ over $x_{0}$ has $\operatorname{dim} U_{x_{0}} \leq n+e-2$, and hence $\operatorname{dim} U \leq 2 n+e-2$. Let $H$ be a general hyperplane in $\mathbb{P}^{N}$. The fibre $U_{H}$ of $U \rightarrow\left(\mathbb{P}^{N}\right)^{\vee}$ over $H$ has $\operatorname{dim} U_{H} \leq n-2$. By Bertini's theorem, $(\operatorname{Sm} X) \cap H \subseteq \operatorname{Sm}(X \cap H)$. This means that for each $x \in$ $\left(\mathfrak{X}_{1} \cap H\right) \backslash U_{H}$ and for each $y \in \operatorname{Sm} \mathcal{D}_{x}$, we have $T_{x}(X) \not \subset H, T_{y}\left(\mathcal{D}_{x}\right) \not \subset H$, and $\operatorname{dim}\left(\operatorname{Sing} \mathcal{D}_{x}\right) \cap H \leq \operatorname{dim} \operatorname{Sing} \mathcal{D}_{x}-1$. This proves the Claim.

Finally we will prove the second part. By the first part, for each $x \in \mathfrak{X}_{1}, \mathcal{D}_{x}=$ $D_{x}(X)$ is an $(n-1)$-dimensional linear subspace. Hence $p_{\mathcal{D}}: \mathcal{D} \rightarrow \mathfrak{X}_{1}$ is generically a $\mathbb{P}^{n-1}$-bundle since the pull-back $\mathcal{L}:=q_{\mathcal{D}}^{*} \mathcal{O}_{X}(1)$ by the second projection $q_{\mathcal{D}}$ : $\mathcal{D} \rightarrow X$ is spanned with $\left.\mathcal{L}\right|_{p_{\mathcal{D}}^{-1}\left(x_{1}\right)}=\mathcal{O}_{D_{x_{1}}(X)}(1)$ for $x_{1} \in \mathfrak{X}_{1}$. We have a rational map $\mathfrak{X}_{1} \rightarrow \mathbb{G}:=\operatorname{Grass}\left(\mathbb{P}^{N}, n-1\right)$ to the Grassmannian variety, whose image has positive dimension. Let $C$ be the normalization of the closure of an irreducible and reduced curve in the image. Let $\mathcal{F}$ be the pull-back of the universal quotient bundle of $\mathbb{G}$ to $C$. The natural morphism $\mathbb{P}_{C}(\mathcal{F}) \rightarrow X$ is birational since $C \rightarrow \mathbb{G}$ gives a birational morphism of $C$ onto its image (see [11, Lemma 1.1]). Note that if $\mathbb{P}_{C}(\mathcal{F}) \rightarrow X$ is not finite, then the intersection of the images of all fibres of $\mathbb{P}_{C}(\mathcal{F}) \rightarrow C$ in $\mathbb{P}^{N}$ is nonempty since each fibre is mapped isomorphically into $X$ and $X$ is a cone and singular since $e \geq 2$. Hence, if $X$ is smooth and $e \geq 2$, $\mathbb{P}_{C}(\mathcal{F}) \rightarrow X$ is finite, and hence isomorphic by the Zariski Main Theorem.

In the proof of Theorem 4.1, the following proposition was used.

Proposition 4.2. Let $X \subseteq \mathbb{P}^{N}$ be a nondegenerate projective surface of codimension e. Suppose $e \geq 2$ and $X$ satisfies $\left(E_{1}\right)$. Then, for a general point $x \in X$, $D_{x}(X)$ is a line.

Proof. By the assumption on $\left(E_{1}\right), D_{x}(X)$ contains a line through $x$. To the contrary, suppose, for general $x \in X$, that $D_{x}(X)$ contains at least two distinct lines. Hence for each $x \in \mathfrak{X}_{1}, D_{x}(X)$ contains two lines, say $L_{x}$ and $L_{x}^{\prime}$. Fix a point $x \in \mathfrak{X}_{1}$. By Lemma 3.4, $L_{x} \cap \mathfrak{X}_{1}$ is a dense open subset of $L_{x}$. Hence, for a general point $x^{\prime}$ of $L_{x}, D_{x^{\prime}}(X)$ also contains two distinct lines $L_{x^{\prime}}$ and $L_{x^{\prime}}^{\prime}$. We may assume $L_{x^{\prime}}=L_{x}$ and $L_{x^{\prime}}^{\prime} \neq L_{x}$. Let $\bar{X}_{L_{x}}$ be the closure of $\pi_{L_{x}}\left(X \backslash L_{x}\right)$ of the linear projection from $L_{x}$. If $L_{x}^{\prime} \cap L_{x^{\prime}}^{\prime} \neq \emptyset$, then $\bar{X}_{L_{x}}$ is a point by the generality of $x^{\prime} \in L_{x}$, and hence $X=\mathbb{P}^{2}$ and $e=0$, a contradiction. Thus $L_{x}^{\prime} \cap L_{x^{\prime}}^{\prime}=\emptyset$. Hence $\bar{X}_{L_{x}}$ is an irreducible curve. Moreover, $\bar{X}_{L_{x}}$ is a line, since there exists a line on $X$ through the general point $x^{\prime \prime}$ of $L_{x}^{\prime}$, different from $L_{x}^{\prime}$ never meeting $L_{x}$ by assumption. Hence $e=1$, a contradiction.

\section{Projective varieties satisfying $\left(E_{2}\right)$}

Lemma 5.1. Let $X \subseteq \mathbb{P}^{N}$ be a nondegenerate projective variety of dimension $n \geq 2$ and codimension $e \geq 3$. Suppose that $X$ satisfies $\left(E_{2}\right)$ but $\operatorname{not}\left(E_{1}\right)$. Then for general points $x_{1}$ and $x_{2}$ of $X$, there exists a smooth conic through $x_{1}$ and $x_{2}$.

Proof. By the assumption on $\left(E_{2}\right)$, there exists an $(n-1)$-dimensional subvariety $D$ of $E_{x_{1} x_{2}}(X)$. Let $z$ be a general point of $D$. Let $C$ be the pure 1-dimensional 
parts of the intersection $\left\langle x_{1}, x_{2}, z\right\rangle \cap D$ with reduced structure. By Lemma 1.2, $C \cap\left\langle x_{1}, x_{2}\right\rangle \subseteq X \cap\left\langle x_{1}, x_{2}\right\rangle=\left\{x_{1}, x_{2}\right\}$. If $C$ contains a line, then the line passes through only one of $x_{1}$ and $x_{2}$, and hence $\operatorname{dim} \pi_{x_{i}}\left(D \backslash\left\{x_{i}\right\}\right) \leq n-2$ for $i=1$ or 2 by the generality of $z \in D$, which means $X$ satisfies $\left(E_{1}\right)$, a contradiction. Therefore $C$ is a smooth conic through $x_{1}$ and $x_{2}$.

Theorem 5.2. Let $X \subseteq \mathbb{P}^{N}$ be a nondegenerate projective variety of dimension $n \geq 2$ and codimension $e \geq 3$. Suppose that $X$ satisfies $\left(E_{2}\right)$ but not $\left(E_{1}\right)$. Then $e=3$ and $X \subseteq \mathbb{P}^{n+3}$ is projectively equivalent to the cone over the Veronese surface $v_{2}\left(\mathbb{P}^{2}\right) \subseteq \mathbb{P}^{5}$ with an $(n-3)$-dimensional vertex. In particular, $X$ is smooth if and only if $n=2$.

Proof. Let $x_{1}$ be general points of $X$. Let $\pi_{x_{1}}: \mathbb{P}^{n+e} \rightarrow \mathbb{P}^{n+e-1}$ be the linear projection from $x_{1}$, where the target $\mathbb{P}^{n+e-1}$ is considered to be a subspace of $\mathbb{P}^{n+e}$ away from $x_{1}$. Let $\bar{X} \subseteq \mathbb{P}^{n+e-1}$ be the closure of $\pi_{x_{1}}\left(X \backslash\left\{x_{1}\right\}\right)$. Note that $\bar{X}$ is nondegenerate since $X$ is so. By our assumption and Lemma 3.2, $\bar{X}$ satisfies $\left(E_{1}\right)$ and hence, by Theorem $4.1, \bar{X} \subseteq \mathbb{P}^{n+e-1}$ is a scroll. First we will construct a projective bundle with a birational morphism to $\bar{X}$ (Steps 1,2), and describe the structure of the projective bundle and $X$ (Steps 3, 4, 5). Next we will write the induced birational rational map from the projective bundle to $X$ (Step 6) and show that $X$ is the cone over the Veronese surface (Step 7).

Step 1. Let $x_{2}, x_{2}^{\prime}$ be general points of $X$. We may assume $\left(x_{1}, x_{2}\right),\left(x_{1}, x_{2}^{\prime}\right) \in \mathfrak{X}_{2} \subseteq$ $X \times X$ (see Lemma 3.4) and $x_{1}, x_{2}, x_{2}^{\prime} \in \operatorname{Sm} X$. Moreover, we may assume that $\bar{x}_{2}:=\pi_{x_{1}}\left(x_{2}\right)$ and $\bar{x}_{2}^{\prime}:=\pi_{x_{1}}\left(x_{2}^{\prime}\right)$ lie on the dense open subset $\overline{\mathfrak{X}}_{1}$ of $\bar{X}$ in Lemma 3.4 with the family $p_{\overline{\mathcal{D}}}: \overline{\mathcal{D}} \rightarrow \overline{\mathfrak{X}}_{1}$. Let $C$ be a smooth conic on $X$ through $x_{2}$ and $x_{2}^{\prime}$ in Lemma 5.1. Let $\bar{C}$ be the closure of $\pi_{x_{1}}\left(C \backslash\left\{x_{1}\right\}\right)$. Let $\mathbb{E}_{x_{1}}$ be the image $\pi_{x_{1}}\left(T_{x_{1}}(X) \backslash\left\{x_{1}\right\}\right)$ of the (embedded) tangent space $T_{x_{1}}(X)$. Consider the linear projection $\pi_{\mathbb{E}_{x_{1}}}: \mathbb{P}^{n+e-1} \rightarrow \mathbb{P}^{e-1}$ from $\mathbb{E}_{x_{1}}$, where the target $\mathbb{P}^{e-1}$ is considered to be a subspace of $\mathbb{P}^{n+e}$ away from $T_{x_{1}}(X)$. Let $\overline{\bar{X}}$ (resp. $\overline{\bar{C}}$ ) be the closure of $\pi_{\mathbb{E}_{x_{1}}}\left(\bar{X} \backslash \mathbb{E}_{x_{1}}\right)$ (resp. $\pi_{\mathbb{E}_{x_{1}}}\left(\bar{C} \backslash \mathbb{E}_{x_{1}}\right)$ ). We claim the following:

(1) For an $(n-1)$-dimensional subvariety $D \subseteq E_{x_{1} x_{2}}(X)$ (resp. $D^{\prime} \subseteq E_{x_{1} x_{2}^{\prime}}(X)$ ) of $X$, the closure $\bar{D}$ (resp. $\left.\bar{D}^{\prime}\right)$ of $\pi_{x_{1}}\left(D \backslash\left\{x_{1}\right\}\right)$ (resp. $\pi_{x_{1}}\left(D^{\prime} \backslash\left\{x_{1}\right\}\right)$ ) is an $(n-1)$-dimensional linear subspace in $\mathbb{P}^{n+e-1}$ passing through $\bar{x}_{2} \in \bar{C}$ (resp. $\bar{x}_{2}^{\prime} \in \bar{C}$ ) and meeting with $\mathbb{E}_{x_{1}}$ in dimension $n-2$.

(2) $\overline{\bar{X}}=\overline{\bar{C}}$ and $\overline{\bar{C}}$ is a conic in $\mathbb{P}^{e-1}$. In particular, $e=3$ and $\bar{C}$ is also conic in $\mathbb{P}^{n+e-1}$ with $\mathbb{E}_{x_{1}} \cap\langle\bar{C}\rangle=\emptyset$, where $\langle\bar{C}\rangle$ is the linear span of $\bar{C}$.

(1) We have only to prove (1) for $D$. By Lemma 3.1, $\bar{x}_{2} \in \bar{D}$. By Remark 3.3, $\bar{D}$ is an $(n-1)$-dimensional subvariety of $E_{\bar{x}_{2}}(\bar{X})$, and hence an $(n-1)$-dimensional linear subspace in $\mathbb{P}^{n+e-1}$ by Theorem 4.1. Moreover, $\operatorname{dim} \mathbb{E}_{x_{1}} \cap \bar{D}=n-2$, since the (projective) tangent cone to $D$ at $x_{1}$ is dimension at least $n-1$ and $D \neq T_{x_{1}}(X)$ by the generality of $x_{2} \in X$.

(2) By (1), $\pi_{\mathbb{E}_{x_{1}}}\left(\bar{D} \backslash \mathbb{E}_{x_{1}}\right)$ is a point. But $\pi_{\mathbb{E}_{x_{1}}}\left(\bar{D} \backslash \mathbb{E}_{x_{1}}\right) \neq \pi_{\mathbb{E}_{x_{1}}}\left(\bar{D}^{\prime} \backslash \mathbb{E}_{x_{1}}\right)$ since $\bar{x}_{2}, \bar{x}_{2}^{\prime} \in \bar{X}$ are general and since $\overline{\bar{X}}$ is not a point by the nondegeneracy of $\overline{\bar{X}}$ in $\mathbb{P}^{e-1}(e-1 \geq 2)$. This means that $\overline{\bar{X}}$ and $\overline{\bar{C}}$ are curves in $\mathbb{P}^{e-1}$ and hence $\overline{\bar{X}}=\overline{\bar{C}}$. Moreover, $\overline{\bar{X}}=\overline{\bar{C}}$ is a conic in $\mathbb{P}^{e-1}$ : Indeed, since $C$ is a smooth conic, $\overline{\bar{X}}=\overline{\bar{C}}$ is either a line or a conic in $\mathbb{P}^{e-1}$, but the former is impossible by the nondegeneracy of $\overline{\bar{X}}$. Hence $e=3$. Consequently $\bar{C} \subseteq \mathbb{P}^{n+2}$ is also a conic with $\mathbb{E}_{x_{1}} \cap\langle\bar{C}\rangle=\emptyset$. 
Step 2: Construction of a projective space bundle birational to $\bar{X}$. Let Cone $\left(\mathbb{E}_{x_{1}}, \bar{C}\right)$ $\subseteq \mathbb{P}^{n+2}$ be the cone over $\bar{C}$ with vertex $\mathbb{E}_{x_{1}}$, which is projectively equivalent to the image of the morphism $\mathbb{P}_{\mathbb{P}^{1}}\left(\mathcal{O}_{\mathbb{P}^{1}}(2) \oplus \mathcal{O}_{\mathbb{P}^{1}}^{\oplus n}\right) \rightarrow \mathbb{P}^{n+2}$ defined by the complete linear system of the tautological line bundle by (2) of Step 1 . Here $\mathcal{O}_{\mathbb{P} 1}^{\oplus n} \cong H^{0}\left(\mathcal{O}_{\mathbb{E}_{x_{1}}}(1)\right) \otimes$ $\mathcal{O}_{\mathbb{P}^{1}}$, and we identify $C$ and $\bar{C}$ with $\mathbb{P}^{1}$. Let $\varphi: \mathbb{P}_{\mathbb{P}^{1}}\left(\mathcal{O}_{\mathbb{P}^{1}}(2) \oplus \mathcal{O}_{\mathbb{P}^{1}}^{\oplus n}\right) \rightarrow \operatorname{Cone}\left(\mathbb{E}_{x_{1}}, \bar{C}\right)$ be the induced birational morphism. We claim that there exists a quotient $\alpha$ : $\mathcal{O}_{\mathbb{P}^{1}}^{\oplus n} \rightarrow \mathcal{G}$ on $\mathbb{P}^{1}(\cong C)$ for a nontrivial locally free sheaf $\mathcal{G}$ of rank $n-1$ such that $\varphi\left(\mathbb{P}_{\mathbb{P}^{1}}\left(\mathcal{O}_{\mathbb{P}^{1}}(2) \oplus \mathcal{G}\right)\right)=\bar{X}$ under the inclusion $\mathbb{P}_{\mathbb{P}^{1}}\left(\mathcal{O}_{\mathbb{P}^{1}}(2) \oplus \mathcal{G}\right) \subseteq \mathbb{P}_{\mathbb{P}^{1}}\left(\mathcal{O}_{\mathbb{P}^{1}}(2) \oplus \mathcal{O}_{\mathbb{P}^{1}}^{\oplus n}\right)$ corresponding to $1 \oplus \alpha: \mathcal{O}_{\mathbb{P}^{1}}(2) \oplus \mathcal{O}_{\mathbb{P}^{1}}^{\oplus n} \rightarrow \mathcal{O}_{\mathbb{P}^{1}}(2) \oplus \mathcal{G}$. Consequently, letting $\varphi^{\prime}: \mathbb{P}_{\mathbb{P}^{1}}\left(\mathcal{O}_{\mathbb{P}^{1}}(2) \oplus \mathcal{G}\right) \rightarrow \bar{X}$ be the induced morphism from $\varphi$, we have the following commutative diagram:

$$
\begin{aligned}
& \underset{\mathbb{P}_{\mathbb{P}^{1}}\left(\mathcal{O}_{\mathbb{P}^{1}}(2) \oplus \mathcal{O}_{\mathbb{P}^{1}}^{\oplus n}\right)}{\cup} \stackrel{\stackrel{\varphi}{\rightarrow}}{\operatorname{Cone}\left(\mathbb{E}_{x_{1}}, \bar{C}\right)} \underset{\cup}{\cup} \mathbb{P}^{n+2} \\
& \mathbb{P}_{\mathbb{P}^{1}}\left(\mathcal{O}_{\mathbb{P}^{1}}(2) \oplus \mathcal{G}\right) \quad \stackrel{\varphi^{\prime}}{\rightarrow} \quad \bar{X} .
\end{aligned}
$$

To this purpose, consider the family $p_{\overline{\mathcal{D}}}: \overline{\mathcal{D}} \rightarrow \overline{\mathfrak{X}}_{1}$, which is a $\mathbb{P}^{n-1}$-bundle by Theorem 4.1. Since $\bar{x}_{2}, \bar{x}_{2}^{\prime} \in \overline{\mathfrak{X}}_{1}$, there exists a dense open subset $C_{0}$ of $C$ such that $\left\{x_{1}\right\} \times C_{0} \subseteq \mathfrak{X}_{2}$ in $X \times X$ and $\pi_{x_{1}}\left(C_{0}\right) \subseteq \overline{\mathfrak{X}}_{1}$. For each $x \in C_{0}$, let $D(x)$ be an $(n-1)$-dimensional subvariety of $E_{x_{1} x}(X)$. By Remark 3.3, the closure $\bar{D}(x)$ of $\pi_{x_{1}}\left(D(x) \backslash\left\{x_{1}\right\}\right)$ is an $(n-1)$-dimensional subvariety of $E_{\bar{x}}(\bar{X})$ for $\bar{x}:=\pi_{x_{1}}(x)$, and hence is the linear subspace $p_{\overline{\mathcal{D}}}^{-1}(\bar{x})$. Thus the pull-back of $p_{\overline{\mathcal{D}}}: \overline{\mathcal{D}} \rightarrow \overline{\mathfrak{X}}_{1}$ by $C_{0} \rightarrow \overline{\mathfrak{X}}_{1}$ is the family $\{\bar{D}(x)\}_{x \in C_{0}}$, and $\bar{X}$ is the closure of $\bigcup_{x \in C_{0}} \bar{D}(x)$ since $\overline{\bar{X}}=\overline{\bar{C}}$. By Lemma 3.1, $\bar{D}(x)$ is through $\bar{x} \in \bar{C}$. Hence $\operatorname{dim} \bar{D}(x) \cap \mathbb{E}_{x_{1}}=n-2$ since $\mathbb{E}_{x_{1}} \cap\langle\bar{C}\rangle=\emptyset$ and since the projective tangent cone to $D(x)$ at $x_{1}$ is of dimension at least $n-1$. Thus we have a rational map from $C(\cong \bar{C})$ to the dual space $\mathbb{E}_{x_{1}}^{\vee}$ to $\mathbb{E}_{x_{1}}\left(\cong \mathbb{P}^{n-1}\right)$ mapping $x \in C_{0}$ to $\bar{D}(x) \cap \mathbb{E}_{x_{1}}$. This map extends to a unique morphism $\gamma: C \rightarrow \mathbb{E}_{x_{1}}^{\vee}$. By pulling back the universal quotient bundle on $\mathbb{E}_{x_{1}}^{\vee}$ by $\gamma: C \rightarrow \mathbb{E}_{x_{1}}^{\vee}$, we have $\alpha$. The nontriviality of $\mathcal{G}$ follows from the nondegeneracy of $\bar{X} \subseteq \mathbb{P}^{n+2}$.

Step 3. Let $\sigma: \hat{X}:=\mathrm{Bl}_{x_{1}}(X) \rightarrow X$ be the blowing-up of $X$ at $x_{1}$. The projection $\pi_{x_{1}, X}: X \rightarrow \mathbb{P}^{n+2}$ extends to the morphism $\hat{\pi}_{x_{1}, X}: \hat{X} \rightarrow \mathbb{P}^{n+2}$. Let $\hat{\pi}_{x_{1}, X}^{\prime}:$ $\hat{X} \rightarrow \bar{X}$ be the induced morphism. Let $p: \mathbb{P}_{\mathbb{P}^{1}}\left(\mathcal{O}_{\mathbb{P}^{1}}(2) \oplus \mathcal{G}\right) \rightarrow \mathbb{P}^{1}$ be the projection. For each $x \in C\left(\cong \mathbb{P}^{1}\right)$, set $F_{x}:=\varphi^{\prime}\left(p^{-1}(x)\right)$. Thus we have:

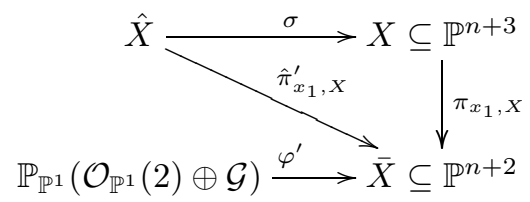

We claim that the morphisms above induce isomorphisms

$$
\text { (5.2.3) } \pi: X \backslash T_{x_{1}}(X) \rightarrow \bar{X} \backslash \mathbb{E}_{x_{1}} \text { and } \varphi^{\prime \prime}: \bar{X} \backslash \mathbb{E}_{x_{1}} \rightarrow \mathbb{P}_{\mathbb{P}^{1}}\left(\mathcal{O}_{\mathbb{P}^{1}}(2) \oplus \mathcal{G}\right) \backslash \mathbb{P}_{\mathbb{P}^{1}}(\mathcal{G}),
$$

and hence these are smooth. In particular, $X$ is smooth along $C, \pi$ is isomorphic along $C$, and $\bar{X}$ is smooth at each $\bar{x}=\pi_{x_{1}}(x) \in \bar{C}$ with $T_{\bar{x}}(\bar{X})=\left\langle T_{\bar{x}}(\bar{C}), F_{x}\right\rangle$.

First $\varphi^{\prime \prime}$ is isomorphic, since $\varphi$ is isomorphic except on $\mathbb{E}_{x_{1}}$ and $\varphi^{-1}\left(\mathbb{E}_{x_{1}}\right)=$ $\mathbb{P}_{\mathbb{P}^{1}}\left(\mathcal{O}_{\mathbb{P}^{1}}^{\oplus n}\right)$, and since $\mathbb{P}_{\mathbb{P}^{1}}\left(\mathcal{O}_{\mathbb{P}^{1}}(2) \oplus \mathcal{G}\right) \cap \mathbb{P}_{\mathbb{P}^{1}}\left(\mathcal{O}_{\mathbb{P}^{1}}^{\oplus n}\right)=\mathbb{P}_{\mathbb{P}^{1}}(\mathcal{G})$ in $\mathbb{P}_{\mathbb{P}^{1}}\left(\mathcal{O}_{\mathbb{P}^{1}}(2) \oplus \mathcal{O}_{\mathbb{P}^{1}}^{\oplus n}\right)$ by Step 2. Hence $\bar{X} \backslash \mathbb{E}_{x_{1}} \subseteq \operatorname{Sm} \bar{X}$. In particular, $\bar{X}$ is smooth at each $\bar{x}=\pi_{x_{1}}(x) \in \bar{C}$ 
with $T_{\bar{x}}(\bar{X})=\left\langle T_{\bar{x}}(\bar{C}), F_{x}\right\rangle$. Moreover, by (1.1.2), $\pi$ is finite and birational by the generality of $x_{1}$. Hence $\pi$ is isomorphic by the Zariski Main Theorem, and consequently $X \backslash T_{x_{1}}(X)$ is smooth.

Step 4. Let $\overline{\mathcal{H}} \subseteq\left|\mathcal{O}_{\mathbb{P}^{n+2}}(1)\right|$ be the linear subsystem of all hyperplanes in $\mathbb{P}^{n+2}$ containing $\langle\bar{C}\rangle$ and let $\mathcal{H} \subseteq\left|\mathcal{O}_{\mathbb{P}^{n+3}}(1)\right|$ be the subsystem of hyperplanes in $\mathbb{P}^{n+3}$ containing $\left\langle C, x_{1}\right\rangle$. Here these are in one-to-one correspondence by the pull-back $\pi_{x_{1}}^{*}$. Let $\bar{H}_{1}, \ldots, \bar{H}_{n-2} \in \overline{\mathcal{H}}$ be general members, and let $H_{1}, \ldots, H_{n-2} \in \mathcal{H}$ be the corresponding (general) members. For each $m(n \geq m \geq 2)$, we take schemetheoretic intersections

$$
\begin{aligned}
& \mathbb{E}_{x_{1}}^{m-1}:=\mathbb{E}_{x_{1}} \cap \bar{H}_{1} \cap \cdots \cap \bar{H}_{n-m}, \quad F_{x}^{m-1}:=F_{x} \cap \bar{H}_{1} \cap \cdots \cap \bar{H}_{n-m}, \\
& \bar{X}^{m}:=\bar{X} \cap \bar{H}_{1} \cap \cdots \cap \bar{H}_{n-m}, \quad X^{m}:=X \cap H_{1} \cap \cdots \cap H_{n-m},
\end{aligned}
$$

and note that $\operatorname{dim} \mathbb{E}_{x_{1}}^{m-1}=m-1$. We claim the following:

(1) For each $x \in C, F_{x} \cap \mathbb{E}_{x_{1}}^{m-1}$ is a hyperplane in $\mathbb{E}_{x_{1}}^{m-1}$, and hence the correspondence $x \mapsto F_{x} \cap \mathbb{E}_{x_{1}}^{m-1}$ defines a morphism $\bar{\gamma}_{m}: C \rightarrow\left(\mathbb{E}_{x_{1}}^{m-1}\right)^{\vee}$, where $\left(\mathbb{E}_{x_{1}}^{m-1}\right)^{\vee}$ is the dual projective space of $\mathbb{E}_{x_{1}}^{m-1}$. Consequently, $\operatorname{dim} F_{x}^{m-1}=$ $m-1$ for all $x \in C$.

(2) $\bar{X}^{m}$ is $m$-dimensional, irreducible, reduced, and smooth outside of $\mathbb{E}_{x_{1}}^{m-1}$. In particular, $\bar{X}^{m}$ is smooth at each $\bar{x}=\pi_{x_{1}}(x) \in \bar{C}$ with $T_{\bar{x}}\left(\bar{X}^{m}\right)=$ $\left\langle T_{\bar{x}}(\bar{C}), F_{x}^{m-1}\right\rangle$.

(3) $X^{m}$ is $m$-dimensional, irreducible, reduced, and smooth at $x_{1}$.

Before proving the claim, we make a few remarks. The rational map defined by $\overline{\mathcal{H}}$ is the projection $\pi_{\langle\bar{C}\rangle}: \mathbb{P}^{n+2} \rightarrow \mathbb{P}^{n-1}$ from $\langle\bar{C}\rangle$ and $\pi_{\langle\bar{C}\rangle}(\bar{X} \backslash\langle\bar{C}\rangle)=$ $\pi_{\langle\bar{C}\rangle}\left(\mathbb{E}_{x_{1}}\right)=\mathbb{P}^{n-1}$. Similarly, the rational map defined by $\mathcal{H}$ is the projection $\pi_{\left\langle C, x_{1}\right\rangle}: \mathbb{P}^{n+3} \rightarrow \mathbb{P}^{n-1}$ from $\left\langle C, x_{1}\right\rangle$ and $\pi_{\left\langle C, x_{1}\right\rangle}\left(X \backslash\left\langle C, x_{1}\right\rangle\right)=\mathbb{P}^{n-1}$. The base locus Bs $\left.\overline{\mathcal{H}}\right|_{\bar{X}}$ is $\langle\bar{C}\rangle \cap \bar{X}$, and $\langle\bar{C}\rangle \cap \bar{X}=\bar{C}$ as sets, since $\langle\bar{C}\rangle \rightarrow\langle\overline{\bar{C}}\rangle$ is isomorphic and $\overline{\bar{X}}=\overline{\bar{C}}$. Also Bs $\left.\mathcal{H}\right|_{X}=\left\langle C, x_{1}\right\rangle \cap X=\pi_{x_{1}, X}^{-1}(\bar{C}) \cup\left\{x_{1}\right\}=C \cup\left\{x_{1}\right\}$, since $\pi$ in (5.2.3) is isomorphic.

(1) For $m=n$, (1) holds by Step 2. By descending induction on $m$, assume (1) for $m+1$ and we will show (1) for $m<n$. Since $\bar{H}_{n-m} \in \overline{\mathcal{H}}$ is general, $\mathbb{E}_{x_{1}}^{m-1}$ as a point of $\left(\mathbb{E}_{x_{1}}^{m}\right)^{\vee}$ is general and hence does not lie on the image of $\bar{\gamma}_{m+1}$. Thus (1) holds for $m$.

(2) For $m=n,(2)$ holds by Step 3. By descending induction on $m$, assume (2) for $m+1$ and we will show (2) for $m<n$. For the linear system $\left.\overline{\mathcal{H}}\right|_{\bar{X}^{m+1}}$ on $\bar{X}^{m+1}$, by the remark above, Bs $\left.\overline{\mathcal{H}}\right|_{\bar{X}}{ }^{m+1}=\langle\bar{C}\rangle \cap \bar{X}^{m+1}=\bar{C}$ and $\pi_{\langle\bar{C}\rangle}\left(\bar{X}^{m+1} \backslash\langle\bar{C}\rangle\right) \cong \mathbb{P}^{m}$. By Bertini's Theorem (see [4. (3.4.8), (3.4.2), (3.4.9), and (3.4.10)]), $\operatorname{dim} \bar{X}^{m}=m$, $\bar{X}^{m}$ is reduced (i.e., satisfies Serre's conditions $\left(S_{1}\right)$ and $\left.\left(R_{0}\right)\right)$ outside of $\bar{C}$ (= Bs $\left.\left.\overline{\mathcal{H}}\right|_{\bar{X}^{m+1}}\right)$, and $\bar{X}^{m}$ is irreducible with $\operatorname{Sing} \bar{X}^{m} \subseteq \mathbb{E}_{x_{1}} \cup \bar{C}$. To see that $\bar{X}^{m}$ is smooth along $\bar{C}$, by the smoothness of $\bar{X}^{m+1}$ along $\bar{C}$, we have to show that $T_{\bar{x}}\left(\bar{X}^{m+1}\right)\left(=\left\langle T_{\bar{x}}(\bar{C}), F_{x}^{m}\right\rangle\right) \nsubseteq \bar{H}_{n-m}$ for each $\bar{x}=\pi_{x_{1}}(x) \in \bar{C}$. But this is true since $F_{x}^{m-1} \subsetneq F_{x}^{m}$ for all $x \in C$ by (1).

(3) By the same way as in (2) using descending induction and Bertini's Theorem, $X^{m}$ is irreducible and satisfies $\left(R_{0}\right)$ and $\left(S_{1}\right)$ outside of Bs $\left.\mathcal{H}\right|_{X}=C \cup\left\{x_{1}\right\}$. By (2), $X^{m}$ is smooth outside of $T_{x_{1}}(X)$, in particular, smooth along $C$, since $X^{m} \backslash T_{x_{1}}(X)$ and $\bar{X}^{m} \backslash \mathbb{E}_{x_{1}}$ are isomorphic by $\pi$ in (5.2.3). Moreover, $X^{m}$ is smooth at $x_{1}$, since $T_{x_{1}}(X) \cap H_{1} \cap \cdots \cap H_{i-1} \not \subset H_{i}$ for each $i \leq n-2$ by the generality of $H_{i} \in \mathcal{H}$ and $\langle\bar{C}\rangle \cap \mathbb{E}_{x_{1}}=\emptyset$. Thus we have (3). 
Step 5. We claim that $\mathcal{G} \cong \mathcal{O}_{\mathbb{P}^{1}}(1) \oplus \mathcal{O}_{\mathbb{P}^{1}}^{\oplus n-2}$.

Since $\bar{\gamma}_{2}: C \rightarrow\left(\mathbb{E}_{x_{1}}^{1}\right)^{\vee}$ in Step 4 is a morphism, the trivial subsheaf $\mathcal{O}_{\mathbb{P} 1}^{\oplus n-2} \subseteq$ $\mathcal{O}_{\mathbb{P}^{1}}^{\oplus n}$ corresponding to $H^{0}\left(\mathcal{I}_{\mathbb{E}_{x_{1}}^{1} / \mathbb{E}_{x_{1}}}(1)\right) \subseteq H^{0}\left(\mathcal{O}_{\mathbb{E}_{x_{1}}}(1)\right)$ can be seen as a subbundle of $\mathcal{G}$ via $\alpha: \mathcal{O}_{\mathbb{P} 1}^{\oplus n} \rightarrow \mathcal{G}$ and its quotient $\mathcal{G} / \mathcal{O}_{\mathbb{P} 1}^{\oplus n-2}$ is the locally free sheaf on $\mathbb{P}^{1}$ which comes from the universal quotient bundle on $\left(\mathbb{E}_{x_{1}}^{1}\right)^{\vee}$ by $\bar{\gamma}_{2}$. Thus, to prove the claim of Step 5 , we have only to show that $\mathcal{G} / \mathcal{O}_{\mathbb{P 1}}^{\oplus n-2} \cong \mathcal{O}_{\mathbb{P}^{1}}(1)$ since $\operatorname{Ext}^{1}\left(\mathcal{O}_{\mathbb{P}^{1}}(1), \mathcal{O}_{\mathbb{P} 1}^{\oplus n-2}\right)=0$. To this purpose, we have only to show that $\bar{\gamma}_{2}$ is generically one-to-one, since $\bar{\gamma}_{2}$ is a nonconstant endomorphism of $\mathbb{P}^{1}$. Suppose to the contrary that for a general $x \in C$ there exists $x^{\prime} \in C$ such that $\bar{\gamma}_{2}(x)=\bar{\gamma}_{2}\left(x^{\prime}\right)$, i.e., $F_{x}^{1} \cap \mathbb{E}_{x_{1}}=F_{x^{\prime}}^{1} \cap \mathbb{E}_{x_{1}}$. Let $\tilde{F}_{x}^{1} \subseteq X^{2}$ (resp. $\tilde{F}_{x^{\prime}}^{1} \subseteq X^{2}$ ) be the strict transform of $F_{x}^{1} \subseteq \bar{X}^{2}$ (resp. $F_{x^{\prime}}^{1} \subseteq \bar{X}^{2}$ ) by the birational map $X^{2} \rightarrow \bar{X}^{2}$ induced from $\pi_{x_{1}, X}^{\prime}$ in (5.2.2). By the generality of $x \in C, \tilde{F}_{x}^{1}$ and $\tilde{F}_{x^{\prime}}^{1}$ pass though $x_{1}$, as we will see below. Let $L \subseteq \mathbb{P}^{n+3}$ be the tangent line to $X^{2}$ at $x_{1}$ with direction corresponding to $F_{x}^{1} \cap \mathbb{E}_{x_{1}}=F_{x^{\prime}}^{1} \cap \mathbb{E}_{x_{1}}$. Let $\Lambda_{0}$ be the linear span $\left\langle L, x, x^{\prime}\right\rangle$ in $\mathbb{P}^{n+3}$. Note that $\Lambda_{0} \not \supset T_{x_{1}}\left(X^{2}\right)$ since $\mathbb{E}_{x_{1}} \cap\langle\bar{C}\rangle=\emptyset$ and $x, x^{\prime} \in C$. Let $H^{\prime}$ be a hyperplane of $\mathbb{P}^{n+3}$ containing $\Lambda_{0}$ but not $T_{x_{1}}\left(X^{2}\right)$. Then $X^{2} \cap H^{\prime}$ is smooth at $x_{1}$. But $X^{2} \cap H^{\prime}$ contains the distinct curves $\tilde{F}_{x}^{1}$ and $\tilde{F}_{x^{\prime}}^{1}$ through $x_{1}$, since $F_{x}$ and $F_{x^{\prime}}$ are lines in $\pi_{x_{1}}\left(\Lambda_{0} \backslash\left\{x_{0}\right\}\right)$ contained in $\pi_{x_{1}}\left(H^{\prime} \backslash\left\{x_{1}\right\}\right)$, a contradiction. Hence $\bar{\gamma}_{2}$ is generically one-to-one.

To conclude Step 5, we have only to show that for all $x \in C_{0}$ except finite points, the strict transform $\tilde{F}_{x}^{1}$ of $F_{x}^{1}$ by $X^{2} \rightarrow \bar{X}^{2}$ passes though $x_{1}$. For each $x \in C_{0}$, set $D(x)^{1}:=D(x) \cap H_{1} \cap \cdots \cap H_{n-2}$, where $D(x)$ is the $(n-1)$-dimensional subvariety of $E_{x_{1} x}(X)$ in Step 2 and $H_{1}, \ldots, H_{n-2}$ are the hyperplanes of $\mathbb{P}^{n+3}$ in Step 4 . Note that $D(x)^{1}$ contains $x_{1}$ and each irreducible component of $D(x)^{1}$ is of dimension $\geq 1$. Since $X^{2} \backslash T_{x_{1}}\left(X^{2}\right) \cong \bar{X}^{2} \backslash \mathbb{E}_{x_{1}}^{1}$ by $(5.2 .3), D(x)^{1}$ contains $\tilde{F}_{x}^{1}$ as a component, and other components, if they exist, are contained in $T_{x_{1}}\left(X^{2}\right)$. Thus we have only to show that the set of points $x \in C_{0}$ for which $D(x)^{1}$ are reducible is a finite set. If $D(x)^{1}$ is not irreducible for some $x \in C_{0}$, then $D(x)^{1} \cap T_{x_{1}}\left(X^{2}\right)$ is the tangent line to $X^{2}$ at $x_{1}$ with direction $F_{x}^{1} \cap \mathbb{E}_{x_{1}}$, since $\pi_{x_{1}}\left(D(x)^{1} \cap T_{x_{1}}\left(X^{2}\right) \backslash\left\{x_{1}\right\}\right) \subseteq F_{x}^{1} \cap \mathbb{E}_{x_{1}}$. Thus these points $x \in C_{0}$ such that $D(x)^{1}$ are reducible are finitely many since $\operatorname{dim} X^{2} \cap T_{x_{1}}\left(X^{2}\right) \leq 1$ by $e=3$, as required.

Step 6 . We claim that the rational map $\hat{\pi}_{x_{1}, X}^{\prime-1} \circ \varphi^{\prime}: \mathbb{P}:=\mathbb{P}_{\mathbb{P}^{1}}\left(\mathcal{O}_{\mathbb{P}^{1}}(2) \oplus \mathcal{O}_{\mathbb{P}^{1}}(1) \oplus\right.$ $\left.\mathcal{O}_{\mathbb{P 1}}^{\oplus n-2}\right) \rightarrow \hat{X}$ is isomorphic in codimension 1 (i.e., $\hat{\pi}_{x_{1}, X}^{\prime-1} \circ \varphi^{\prime}$ defines an isomorphism outside of closed subsets of codimension at least two) and that the composite $\mathbb{P} \rightarrow \mathbb{P}^{n+3}$ of $\sigma \circ \hat{\pi}_{x_{1}, X}^{\prime-1} \circ \varphi^{\prime}$ and the embedding $X \rightarrow \mathbb{P}^{n+3}$ is defined by the complete linear system $\left|\mathcal{O}_{\mathbb{P}}(2) \otimes p^{*} \mathcal{O}_{\mathbb{P}^{1}}(-2)\right|$ for the tautological line bundle $\mathcal{O}_{\mathbb{P}}(1)$ on $\mathbb{P}$.

The composite $\mathbb{P} \rightarrow \mathbb{P}^{n+2}$ of $\varphi^{\prime}$ and the inclusion $\bar{X} \subseteq \mathbb{P}^{n+2}$ is defined by $\left|\mathcal{O}_{\mathbb{P}}(1)\right|$ since $\bar{X}$ is nondegenerate in $\mathbb{P}^{n+2}$. Hence $\bar{X}$ is a cone with vertex $\Lambda:=$ $\varphi^{\prime}\left(\mathbb{P}_{\mathbb{P}^{1}}\left(\mathcal{O}_{\mathbb{P}^{1}}^{\oplus n-2}\right)\right)$ and $\varphi^{\prime}$ is isomorphic in codimension one (precisely $\mathbb{P} \backslash \mathbb{P}_{\mathbb{P}^{1}}\left(\mathcal{O}_{\mathbb{P}^{1}}^{\oplus n-2}\right)$ $\rightarrow \bar{X} \backslash \Lambda$ is isomorphic) and $\operatorname{Sing} \bar{X}=\Lambda\left(\subseteq \mathbb{E}_{x_{1}}\right)$. On the other hand, by the assumption on $\left(E_{1}\right)$ for $X$, the morphism $\hat{\pi}_{x_{1}, X}^{\prime}$ has no exceptional $(n-1)$ dimensional subvariety of $\hat{X}$ by Lemma 1.3 . Hence $\hat{\pi}_{x_{1}, X}^{\prime}$ is proper, birational, and finite in codimension one, and consequently, $\hat{\pi}_{x_{1}, X}^{\prime}$ is isomorphic in codimension one by the Zariski Main Theorem, since $\operatorname{dim} \operatorname{Sing} \bar{X}=n-3$. Thus $\hat{\pi}_{x_{1}, X}^{-1} \circ \varphi^{\prime}$ is isomorphic in codimension 1. Under $\hat{\pi}_{x_{1}, X}^{\prime-1} \circ \varphi^{\prime}$, the exceptional divisor $E$ of 
$\sigma: \hat{X} \rightarrow X$ corresponds to $\tilde{\mathbb{E}}_{x_{1}}:=\mathbb{P}_{\mathbb{P}^{1}}\left(\mathcal{O}_{\mathbb{P}^{1}}(1) \oplus \mathcal{O}_{\mathbb{P}^{1}}^{\oplus n-2}\right) \subseteq \mathbb{P}$, and the pull-back $\hat{\pi}_{x_{1}, X}^{\prime *} \mathcal{O}_{\bar{X}}(1)$ corresponds to $\mathcal{O}_{\mathbb{P}}(1)$, since $E=\hat{\pi}_{x_{1}, X}^{\prime *}\left(\mathbb{E}_{x_{1}}\right), \tilde{\mathbb{E}}_{x_{1}}=\varphi^{\prime *}\left(\mathbb{E}_{x_{1}}\right)$, and $\mathcal{O}_{\mathbb{P}}(1)=\varphi^{*} \mathcal{O}_{\bar{X}}(1)$ as divisors. Hence $\sigma^{*} \mathcal{O}_{X}(1)$ corresponds to $\mathcal{O}_{\mathbb{P}}(2) \otimes p^{*} \mathcal{O}_{\mathbb{P}^{1}}(-2)$, since $\hat{\pi}_{x_{1}, X}^{\prime *} \mathcal{O}_{\bar{X}}(1) \cong \sigma^{*} \mathcal{O}_{X}(1) \otimes \mathcal{O}_{\hat{X}}(-E)$ and $\mathcal{O}_{\mathbb{P}}\left(\tilde{\mathbb{E}}_{x_{1}}\right) \cong \mathcal{O}_{\mathbb{P}}(1) \otimes p^{*} \mathcal{O}_{\mathbb{P}^{1}}(-2)$. Note that $\operatorname{dim} H^{0}\left(\mathbb{P}, \mathcal{O}_{\mathbb{P}}(2) \otimes p^{*} \mathcal{O}_{\mathbb{P}^{1}}(-2)\right)=n+4$. Hence $\mathbb{P} \rightarrow \mathbb{P}^{n+3}$ is defined by $\left|\mathcal{O}_{\mathbb{P}}(2) \otimes p^{*} \mathcal{O}_{\mathbb{P}^{1}}(-2)\right|$ since $X \subseteq \mathbb{P}^{n+3}$ is nondegenerate.

Step 7 . We claim that $X$ is projectively equivalent to the cone over $v_{2}\left(\mathbb{P}^{2}\right) \subseteq \mathbb{P}^{5}$.

By Step $6, X \subseteq \mathbb{P}^{n+3}$ is the closure of the image of the birational map $\mathbb{P} \rightarrow \mathbb{P}^{n+3}$ defined by $\left|\mathcal{O}_{\mathbb{P}}(2) \otimes p^{*} \mathcal{O}_{\mathbb{P}^{1}}(-2)\right|$. Hence we will show that the closure of the image of $\mathbb{P}$ is the cone Cone $\left(\Lambda, v_{2}\left(\mathbb{P}^{2}\right)\right)$ over $v_{2}\left(\mathbb{P}^{2}\right)$ with vertex $\Lambda=\varphi^{\prime}\left(\mathbb{P}_{\mathbb{P}^{1}}\left(\mathcal{O}_{\mathbb{P} 1}^{\oplus n-2}\right)\right)$ in $\mathbb{P}^{n+3}$. To this purpose, we take homogeneous coordinates $s, t$ of $\mathbb{P}^{1}$ and a formal basis $Z_{1}, \ldots, Z_{n}$ of $\mathcal{O}_{\mathbb{P}^{1}}(2) \oplus \mathcal{O}_{\mathbb{P}^{1}}(1) \oplus \mathcal{O}_{\mathbb{P}^{1}}^{\oplus n-2}$ (i.e., $\mathcal{O}_{\mathbb{P}^{1}}(2) Z_{1} \oplus \mathcal{O}_{\mathbb{P}^{1}}(1) Z_{2} \oplus \bigoplus_{i=3}^{n} \mathcal{O}_{\mathbb{P}^{1}} Z_{i}$ ) so that the $\mathbb{k}$-vector space $H^{0}\left(\mathcal{O}_{\mathbb{P}}(2) \otimes p^{*} \mathcal{O}_{\mathbb{P}^{1}}(-2)\right)$ has a basis

$$
\left(s Z_{1}\right)^{2},\left(s Z_{1}\right)\left(t Z_{1}\right),\left(t Z_{1}\right)^{2},\left(s Z_{1}\right) Z_{2},\left(t Z_{1}\right) Z_{2}, Z_{2}^{2}, Z_{1} Z_{3}, \ldots, Z_{1} Z_{n} \text {. }
$$

These are the pull-backs of the homogeneous coordinates of $\mathbb{P}^{n+3}$ by $\mathbb{P} \rightarrow-\mathbb{P}^{n+3}$, after a suitable coordinate change of $\mathbb{P}^{n+3}$. In the graded ring

$$
\begin{aligned}
& \bigoplus_{m \geq 0} H^{0}\left(\mathbb{P}, \mathcal{O}_{\mathbb{P}}(2 m) \otimes p^{*} \mathcal{O}_{\mathbb{P}^{1}}(-2 m)\right) \\
& \cong \bigoplus_{m \geq 0} H^{0}\left(\mathbb{P}^{1}, \mathcal{O}_{\mathbb{P}^{1}}(-2 m) \otimes \operatorname{Sym}^{m}\left(\mathcal{O}_{\mathbb{P}^{1}}(2) Z_{1} \oplus \mathcal{O}_{\mathbb{P}^{1}}(1) Z_{2} \oplus \bigoplus_{i=3}^{n} \mathcal{O}_{\mathbb{P}^{1}} Z_{i}\right)\right),
\end{aligned}
$$

$\left(s Z_{1}\right)^{2},\left(s Z_{1}\right)\left(t Z_{1}\right),\left(t Z_{1}\right)^{2},\left(s Z_{1}\right) Z_{2},\left(t Z_{1}\right) Z_{2}, Z_{2}^{2}$ have the same relations as the defining equations of the Veronese surface $v_{2}\left(\mathbb{P}^{2}\right)$ in $\mathbb{P}^{5}$, and $Z_{1} Z_{3}, \ldots, Z_{1} Z_{n}$ are algebraically independent. Therefore the closure of the image is projectively equivalent to the cone Cone $\left(\Lambda, v_{2}\left(\mathbb{P}^{2}\right)\right)$ over $v_{2}\left(\mathbb{P}^{2}\right)$ with $(n-3)$-dimensional vertex $\Lambda$ in $\mathbb{P}^{n+3}$, as required.

\section{Projective varieties satisfying $\left(E_{m}\right)(m \geq 3)$ AND THE PROOF OF THEOREM 4}

Theorem 6.1. Let $X \subseteq \mathbb{P}^{N}$ be a nondegenerate projective variety $X \subseteq \mathbb{P}^{N}$ of dimension $n \geq 2$ and codimension $e \geq 4$. For any $m$ with $e-1>m \geq 3$, it does not occur that $X$ satisfies $\left(E_{m}\right)$ but not $\left(E_{m-1}\right)$. Consequently $X$ satisfies $\left(E_{m}\right)$ for some $m$ with $e-1 \geq m \geq 3$ if and only if $X$ satisfies $\left(E_{1}\right)$.

Proof. To the contrary suppose that $X$ satisfies $\left(E_{m}\right)$ but not $\left(E_{m-1}\right)$ for some $m$ $(e-1 \geq m \geq 3)$. Let $x_{1}, \ldots, x_{m-2} \in X$ be general points. Consider the linear projection $\pi_{\Lambda^{\prime}}: \mathbb{P}^{N} \rightarrow \mathbb{P}^{N-m+2}$ from the linear $\operatorname{span} \Lambda^{\prime}:=\left\langle x_{1}, \ldots, x_{m-2}\right\rangle$. Let $\bar{X}_{\Lambda^{\prime}}$ be the closure of $\pi_{\Lambda^{\prime}}\left(X \backslash \Lambda^{\prime}\right)$. By Lemma 3.2, $\bar{X}_{\Lambda^{\prime}}$ satisfies $\left(E_{2}\right)$ but not $\left(E_{1}\right)$. By Theorem 5.2, $\bar{X}_{\Lambda^{\prime}}$ is projectively equivalent to the cone over the Veronese surface $v_{2}\left(\mathbb{P}^{2}\right) \subseteq \mathbb{P}^{5}$ with $(n-3)$-dimensional vertex in $\mathbb{P}^{n+3}$. Consequently $\bar{X}_{\Lambda^{\prime}}$ contains no $(n-1)$-dimensional linear subspace since $v_{2}\left(\mathbb{P}^{2}\right)$ contains no line. But $\bar{X}_{\Lambda^{\prime}}$ contains the images of the embedded tangent spaces $T_{x_{1}}(X), \ldots, T_{x_{m-2}}(X)$, which are linear subspaces of dimension $n-1$, a contradiction. To see the second part, suppose $X$ satisfies $\left(E_{m}\right)$ for some $m(e-2 \geq m \geq 3)$. By the first part, $X$ satisfies $\left(E_{m-1}\right)$. By the same way, $X$ satisfies $\left(E_{m^{\prime}}\right)$ for all $m^{\prime}$ with $m \geq m^{\prime} \geq 2$. 
Hence $X$ satisfies $\left(E_{1}\right)$ by Theorem 5.2 and $e \geq 4$. The converse is obvious by definition.

Now Theorem 3 is a consequence of Theorems 4.1, 5.2, and 6.1.

Proof of Theorem 4. If $X$ is projectively equivalent to none of (3), then $\operatorname{dim} \mathcal{C}(X) \leq$ 0 by Theorem 2. Moreover, if $X$ is projectively equivalent to none of (1) and (2), then $X$ does not satisfy $\left(E_{m}\right)$ for $1 \leq m \leq e-1$ by Theorem 3 . Hence, by Theorem 1 for $m=e-1$, we have the required result.

Remark 6.2. Theorem 4 is an application of Theorem 1 for $m=e-1$. An application of Theorem 1 for $2 \leq m<e-1$ is included in Theorem 4 . On the other hand, an application of Theorem 1 for $m=1$ to the case (2) in Theorem 4 is obtained directly (see Example $6.3(2)$ ).

Example 6.3. We will briefly look at the cases excluded from Theorem 4.

(1) Let $X \subseteq \mathbb{P}^{N}$ be a scroll $\mathbb{P}_{C}(\mathcal{F}) \subseteq \mathbb{P}^{N}$ over a smooth projective curve $C$ for a locally free sheaf $\mathcal{F}$ of rank $n$ on $C$. Let $p: \mathbb{P}_{C}(\mathcal{F}) \rightarrow C$ be the projection. Then $\omega_{X}=\mathcal{O}_{\mathbb{P}_{C}(\mathcal{F})}(-n) \otimes p^{*}\left(\operatorname{det} \mathcal{F} \otimes \omega_{C}\right), \mathcal{O}_{X}(1)=\mathcal{O}_{\mathbb{P}_{C}}(\mathcal{F})$ and $\operatorname{deg} X=c_{1}(\mathcal{F})$. Suppose that $C \cong \mathbb{P}^{1}$ and $\mathcal{F}$ contains $\mathcal{O}_{\mathbb{P}^{1}}(1)$ as a direct summand. Then $\mathcal{O}_{X}(d-$ $n-2) \otimes \omega_{X}^{\vee}=\mathcal{O}_{X}\left(c_{1}-2\right) \otimes p^{*}\left(\omega_{C}^{\vee} \otimes \operatorname{det} \mathcal{F}^{\vee}\right)=\mathcal{O}_{X}\left(c_{1}-2\right) \otimes p^{*}\left(\mathcal{O}_{\mathbb{P}^{1}}\left(2-c_{1}\right)\right)$. Hence it is trivial along the subbundle $\mathbb{P}_{\mathbb{P}^{1}}\left(\mathcal{O}_{\mathbb{P}^{1}}(1)\right)$ of $\mathbb{P}_{\mathbb{P}^{1}}(\mathcal{F})$.

(2) Let $X \subseteq \mathbb{P}^{5}$ be the Veronese surface $v_{2}\left(\mathbb{P}^{2}\right)$ in $\mathbb{P}^{5}$. Hence $\mathcal{O}_{X}(1) \cong \mathcal{O}_{\mathbb{P}^{2}}(2)$, $d=4$, and $e=3$. Consequently $\mathcal{O}_{X}(d-n-e-1) \otimes \omega_{X}^{\vee}=\mathcal{O}_{\mathbb{P}^{2}}(-1)$ is not base-point-free, but $\mathcal{O}_{X}(d-n-e) \otimes \omega_{X}^{\vee}=\mathcal{O}_{\mathbb{P}^{2}}(1)$ is very ample.

(3) Let $X \subseteq \mathbb{P}^{N}$ be a nondegenerate smooth projective variety which is a birational-divisor of type $(\mu, 1)$ of a conical rational scroll for some integer $\mu \geq 2$ as in the definition before Theorem 2. We keep the notation there. Since $X$ is smooth, $\mathcal{O}_{X}(1) \cong \mathcal{O}_{\mathbf{E}_{\mathcal{E}}^{L}}(1) \mid \tilde{X}, d=\operatorname{deg} X=c_{1}(\mathcal{E}) \cdot \mu+1$, and $\omega_{X} \cong \omega_{\tilde{X}}=$ $\mathcal{O}_{\mathbf{E}_{\mathcal{E}}^{L}}(\mu-n-1) \otimes p^{*} \mathcal{O}_{\mathbb{P}^{1}}\left(c_{1}-1\right)$. Set $\hat{L}:=\tilde{X} \cap \mathbb{P}_{\mathbb{P}^{1}}\left(\mathcal{O}_{\mathbb{P}^{1}}^{\oplus 2}\right) \subseteq \mathbb{P}$. By construction, $\hat{L}$ is a divisor of $L \times \mathbb{P}^{2} \cong \mathbb{P}_{\mathbb{P}^{1}}\left(\mathcal{O}_{\mathbb{P}^{1}}^{\oplus 2}\right)$, and $\mathcal{O}_{\mathbb{P}}(1) \mid \hat{L}=\mathcal{O}_{\hat{L}}(1) \cong \mathcal{O}_{L}(1)$ and $p^{*} \mathcal{O}_{\mathbb{P}^{1}}(1) \mid \hat{L}=\mathcal{O}_{\hat{L}}(\mu) \cong \mathcal{O}_{L}(\mu)$. Then $\mathcal{O}_{X}(d-n-2) \otimes \omega_{X}^{\vee} \cong \mathcal{O}_{\tilde{X}}(d-n-2) \otimes$ $\omega_{\tilde{X}}^{\vee}=\left.\mathcal{O}_{\mathbb{P}}\left(\left(c_{1}-1\right) \mu\right) \otimes p^{*} \mathcal{O}_{\mathbb{P}^{1}}\left(1-c_{1}\right)\right|_{\tilde{X}}$, which is trivial along $\hat{L}$ and $L$. Hence $\left|\mathcal{O}_{X}(d-m-n-2) \otimes \omega_{X}^{\vee}\right|$ for $m \geq 1$ has a base locus along $L$.

Example 6.4. In Theorem 1, we have shown that the ramification divisor of some generic inner projection of $X$ is a member of $\left|\mathcal{O}_{X}(d-m-n-2) \otimes \omega_{X}^{\vee}\right|$. Hence its support contains the $(n-1)$-dimensional components of the nonisomorphic locus of the corresponding inner projection. But it does not necessarily contain the $l$ dimensional components for $l \leq n-2$ : Let $X \subseteq \mathbb{P}^{9}$ be the 2-uple embedding $v_{2}\left(\mathbb{P}^{3}\right)$. Hence $\mathcal{O}_{X}(1)=\mathcal{O}_{\mathbb{P}^{3}}(2), d=8$, and $e=6$. Set $m=e-1=5$. Then $\mathcal{O}_{X}(d-n-e-1) \otimes \omega_{X}^{\vee}=\mathcal{O}_{X}$, and hence it has no nonzero effective member. On the other hand, the projection $\pi_{\Lambda, X}: X \backslash \Lambda \rightarrow \mathbb{P}^{4}$ from the linear span $\Lambda:=\left\langle x_{1}, \ldots, x_{5}\right\rangle$ of general points $x_{1}, \ldots, x_{5} \in X$ maps the conics $C_{x_{i} x_{j}}$ on $X$ through $x_{i}$ and $x_{j}$ to points.

\section{Applications}

Proof of Corollary 5. If $e=1$, the proof is clear. Hence assume $e \geq 2$. If $X$ is projectively equivalent to none of (1)-(3) in Theorem 4 , then $\mathcal{O}_{X}(d-n-e) \otimes \omega_{X}^{\vee}$ 
is ample by Theorem 4, and hence, by the Kodaira Vanishing Theorem,

$$
H^{i}\left(X, \mathcal{O}_{X}(j)\right)=0 \quad \text { for } 1 \leq i \leq n \text { and } j \geq d-e-n,
$$

which means $\mathcal{O}_{X}$ is $(d-e)$-regular. When $X$ is the Veronese surface $v_{2}\left(\mathbb{P}^{2}\right) \subseteq \mathbb{P}^{5}$, for $i=1$ and 2 , we obtain that $2(d-e-i) \geq-2$ and

$$
H^{i}\left(X, \mathcal{O}_{X}(d-e-i)\right)=H^{i}\left(\mathbb{P}^{2}, \mathcal{O}_{\mathbb{P}^{2}}(2(d-e-i))\right)=0,
$$

which means $\mathcal{O}_{X}$ is $(d-e)$-regular. Finally suppose that $X$ is a birational-divisor of type $(\mu, 1)$ of a conical rational scroll for some integer $\mu \geq 2$, and we will show that $\mathcal{O}_{X}$ is $(d-e)$-regular. Keep the notation as in the definition before Theorem 2 . Note that $X \cong \tilde{X}$. We have to show that $H^{i}\left(\tilde{X}, \mathcal{O}_{\tilde{X}}(d-e-i)\right)=0$ for $1 \leq i \leq n$. By the exact sequence

$$
0 \rightarrow \mathcal{O}_{\mathbf{E}_{\mathcal{E}}^{L}}(d-e-i-\mu) \otimes p^{*} \mathcal{O}_{\mathbb{P}^{1}}(-1) \rightarrow \mathcal{O}_{\mathbf{E}_{\mathcal{E}}^{L}}(d-e-i) \rightarrow \mathcal{O}_{\tilde{X}}(d-e-i) \rightarrow 0,
$$

we have only to show that

$H^{i}\left(\mathcal{O}_{\mathbf{E}_{\mathcal{E}}^{L}}(d-e-i)\right)=H^{i+1}\left(\mathcal{O}_{\mathbf{E}_{\mathcal{E}}^{L}}(d-e-i-\mu) \otimes p^{*} \mathcal{O}_{\mathbb{P}^{1}}(-1)\right)=0 \quad$ for $\quad 1 \leq i \leq n$.

Since $\operatorname{dim} H^{0}\left(\mathcal{O}_{\mathbf{E}_{\mathcal{E}}^{L}}(1)\right)-1=c_{1}(\mathcal{E})+n \geq N, c_{1}(\mathcal{E}) \geq e \geq 2$, and $d=\operatorname{deg} \tilde{X}=$ $c_{1}(\mathcal{E}) \cdot \mu+1$, we have

$$
d-e-\mu-i \geq\left(c_{1}(\mathcal{E})-1\right)(\mu-1)-i \geq 1-i \geq 1-n .
$$

Consequently, for $k \geq 1$, we have

$$
R^{k} p_{*} \mathcal{O}_{\mathbf{E}_{\mathcal{E}}^{L}}(d-e-i)=0 \quad \text { and } \quad R^{k} p_{*}\left(\mathcal{O}_{\mathbf{E}_{\mathcal{E}}^{L}}(d-e-i-\mu) \otimes p^{*} \mathcal{O}_{\mathbb{P}^{1}}(-1)\right)=0 .
$$

By Leray's spectral sequence, for $1 \leq i \leq n$, we have

$$
\begin{aligned}
H^{i}\left(\mathcal{O}_{\mathbf{E}_{\mathcal{E}}^{L}}(d-e-i)\right) \cong H^{i}\left(\operatorname{Sym}^{d-e-i}\left(\mathcal{O}_{\mathbb{P}^{1}}^{\oplus 2} \oplus \mathcal{E}\right)\right)=0, & \text { and } \\
H^{i+1}\left(\mathcal{O}_{\mathbf{E}_{\mathcal{E}}^{L}}(d-e-i-\mu)\right. & \left.\otimes p^{*} \mathcal{O}_{\mathbb{P}^{1}}(-1)\right) \\
& \cong H^{i+1}\left(\operatorname{Sym}^{d-e-i-\mu}\left(\mathcal{O}_{\mathbb{P}^{1}}^{\oplus 2} \oplus \mathcal{E}\right) \otimes \mathcal{O}_{\mathbb{P}^{1}}(-1)\right)=0,
\end{aligned}
$$

as required.

As another application of Theorem 4 , we study Property $\left(N_{p}\right)$. We recall the definition (see [2] and [8] for the definition and properties): For a very ample line bundle $L$ over a projective variety $X$, consider the embedding $X \rightarrow \mathbb{P}:=$ $\mathbb{P}\left(H^{0}(X, L)\right)$ by $|L|$ and the graded ring $R_{L}:=\bigoplus_{m \geq 0} H^{0}\left(X, L^{\otimes m}\right)$ which is an algebra over a homogeneous coordinate ring $S$ of $\mathbb{P}$ (i.e., $S$ is a polynomial ring of $\operatorname{dim}_{\mathbb{k}} H^{0}(X, L)$ variables). Let $\cdots \rightarrow F_{i} \rightarrow \cdots \rightarrow F_{1} \rightarrow F_{0} \rightarrow R_{L} \rightarrow 0$ ( $F_{i}=$ $\bigoplus_{j} S\left(-a_{i j}\right)$ ) be a minimal free resolution of $R_{L}$ over $S$. We say that $L$ satisfies $\left(N_{0}\right)$ if $F_{0}=S$. For $p \geq 1$, we say that $L$ satisfies $\left(N_{p}\right)$ if $F_{0}=S$ and $F_{i} \cong \bigoplus S(-i-1)$ (i.e., $a_{i j}=i+1$ for all $j$ ) for all $1 \leq i \leq p$.

Corollary 7.1. Let $X \subseteq \mathbb{P}^{N}$ be a nondegenerate smooth projective variety of $d i$ mension $n \geq 2$, codimension e, and degree $d$. Suppose that $X$ is projectively equivalent to none of (1)-(3) in Theorem 4 . Then $\mathcal{O}_{X}(k)$ satisfies Property $\left(N_{k-d+e}\right)$ (if $k-d+e \geq 0)$.

Proof. This is an immediate consequence of a criterion ([2, Corollary 2.2]) for Property $\left(N_{p}\right)$ (see [2, Proof of Proposition 3.3]), which asserts that for a very ample line bundle $A$ on $X$, for a nef line bundle $B$ on $X$, and for an integer $p \geq 0$, setting $l:=n+1+p, L_{l}:=K_{X}+l A+B$ satisfies $\left(N_{p}\right)$. In our situation, we set $A:=\mathcal{O}_{X}(1)$, 
and $B:=\mathcal{O}_{X}(d-n-e-1) \otimes \omega_{X}^{\vee}$, and note that $B$ is semiample (and hence nef) by Theorem 4 . By the above criterion, $L_{l}=\mathcal{O}_{X}(l+d-n-e-1)=\mathcal{O}_{X}(d-e+p)$ satisfies $\left(N_{p}\right)$ if $p \geq 0$. This means for $k:=d-e+p$ that $L_{l}=\mathcal{O}_{X}(k)$ satisfies $\left(N_{k-d+e}\right)$ if $k-d+e \geq 0$.

Remark 7.2. The corollary above improves slightly the result of Ein and Lazarsfeld ([2, Proposition 3.3]): They proved that $\mathcal{O}_{X}(k)$ satisfies Property $\left(N_{k-d+1}\right)$ (if $k-d+1 \geq 0)$ for a smooth projective variety $X \subseteq \mathbb{P}^{N}$ of degree $d$.

Another application of Theorem 4 is the inequalities for the $\Delta$-genus and sectional genus (for the definition and properties, see [6]). For a very ample line bundle $L$ on a smooth projective variety $X$ with canonical line bundle $\omega_{X}$, recall that the $\Delta$-genus $\Delta(X, L)$ and the sectional genus $g(X, L)$ are defined by

$\Delta(X, L)=n+\operatorname{deg} L-\operatorname{dim}_{\mathbb{k}} H^{0}(X, L)$ and $g(X, L)=\frac{1}{2}\left(\omega_{X} \otimes L^{\otimes n-1} \cdot L^{n-1}\right)+1$.

On the other hand, for a nondegenerate projective variety $X \subseteq \mathbb{P}^{N}$ of dimension $n$, codimension $e$, and degree $d$, set $\Delta\left(X, \mathbb{P}^{N}\right):=d-e-1$, which satisfies $\Delta\left(X, \mathbb{P}^{N}\right) \geq$ $\Delta\left(X, \mathcal{O}_{X}(1)\right)$, and the equality holds if $X$ is embedded by the complete linear system $\left|\mathcal{O}_{X}(1)\right|$.

Corollary 7.3. Let $X \subseteq \mathbb{P}^{N}$ be a nondegenerate smooth projective variety of dimension $n \geq 2$, codimension e, and degree $d$. Suppose that $X$ is projectively equivalent to none of (1)-(3) in Theorem 4.

(1) If $\kappa(X) \geq 0$ (i.e., $H^{0}\left(X, \omega_{X}^{\otimes l}\right) \neq 0$ for some $\left.l>0\right)$, then $\Delta\left(X, \mathbb{P}^{N}\right) \geq n$.

(2) $\Delta\left(X, \mathbb{P}^{N}\right) \geq \frac{2}{d}\left(g\left(X, \mathcal{O}_{X}(1)\right)-1\right)+1$.

Proof. By Theorem $4, \mathcal{O}_{X}(d-n-e-1) \otimes \omega_{X}^{\vee}$ has a global section and hence, for the intersection number, we have

$$
\left(\mathcal{O}_{X}(d-n-e-1) \otimes \omega_{X}^{\vee} \cdot \mathcal{O}_{X}(1)^{n-1}\right) \geq 0 .
$$

Hence if $\kappa(X) \geq 0$, then $\left(\omega_{X} \cdot \mathcal{O}_{X}(1)^{n-1}\right) \geq 0$ and hence $d(d-n-e-1) \geq 0$. This means $\Delta\left(X, \mathbb{P}^{N}\right) \geq n$ if $\kappa(X) \geq 0$. For $(2)$, since $\mathcal{O}_{X}(d-n-e-1) \otimes \omega_{X}^{\vee}=$ $\mathcal{O}_{X}(d-e-2) \otimes\left(\omega_{X} \otimes \mathcal{O}_{X}(n-1)\right)^{\vee}$, from (7.3.1) it follows that

$$
d\left(\Delta\left(X, \mathbb{P}^{N}\right)-1\right)-\left(2 g\left(X, \mathcal{O}_{X}(1)\right)-2\right) \geq 0,
$$

and hence $\Delta\left(X, \mathbb{P}^{N}\right) \geq \frac{2}{d}\left(g\left(X, \mathcal{O}_{X}(1)\right)-1\right)+1$, as required.

Remark 7.4. From [7, Theorem 4.4], it follows that

$$
\Delta\left(X, \mathcal{O}_{X}(1)\right) \geq \frac{n}{n+1}\left(\mathcal{O}_{X}(1)^{n}-1\right)
$$

if $\operatorname{dim}_{\mathbb{k}} H^{n}\left(X, \mathcal{O}_{X}\right)=\operatorname{dim}_{\mathbb{k}} H^{0}\left(X, \omega_{X}\right)>0$. Hence, if $\mathcal{O}_{X}(1)^{n} \geq n+3$, this inequality is better than that in (1) of Corollary 7.3.

Remark 7.5. Let $X \subseteq \mathbb{P}^{N}$ be a nondegenerate smooth projective variety, and let $X \subseteq \mathbb{P}^{\tilde{N}}:=\mathbb{P}\left(H^{0}\left(X, \mathcal{O}_{X}(1)\right)\right)$ be the embedding of $X$ by $\left|\mathcal{O}_{X}(1)\right|$. Then $X \subseteq \mathbb{P}^{N}$ is projectively equivalent to a scroll if and only if so is $X \subseteq \mathbb{P}^{\tilde{N}}$. Similarly $X \subseteq \mathbb{P}^{N}$ is projectively equivalent to (3) of Theorem 4 if and only if so is $X \subseteq \mathbb{P}^{\tilde{N}}$. This is because $X \subseteq \mathbb{P}^{N}$ is obtained by the linear projection of $X \subseteq \mathbb{P}^{\tilde{N}}$ from a linear subspace of $\mathbb{P}^{\tilde{N}}$ disjoint from $X$, by taking into account that $H^{0}\left(X, \mathcal{O}_{X}(1)\right) \cong$ $H^{0}\left(\mathbf{E}_{\mathcal{E}}^{L}, \mathcal{O}_{\mathbf{E}_{\mathcal{E}}^{L}}(1)\right)$ for the latter. 
Corollary 7.6. Let $X \subseteq \mathbb{P}^{N}$ be a nondegenerate smooth projective variety of dimension $n \geq 2$, codimension $e$, and degree $d$. Suppose $X$ is projectively equivalent to neither (1) nor (3) of Theorem 4.

(1) If $\Delta\left(X, \mathcal{O}_{X}(1)\right) \leq n$, then $\omega_{X}^{\vee}$ has at most finite base points and hence $\omega_{X}^{\vee}$ is semisimple.

(2) If $\Delta\left(X, \mathcal{O}_{X}(1)\right)<n$, then $\omega_{X}^{\vee}$ is very ample on $X \backslash \mathcal{C}(X)$ and ample on $X$.

Proof. By Remark 7.5, we may assume that $X \subseteq \mathbb{P}^{N}$ is embedded by the complete linear system. If $X$ is the Veronese surface $v_{2}\left(\mathbb{P}^{2}\right)$ in $\mathbb{P}^{5}$, the assertion is clear since $\Delta\left(X, \mathcal{O}_{X}(1)\right)=0$ and $\omega_{X}^{\vee}=\mathcal{O}_{\mathbb{P}^{2}}(3)$. Thus suppose $X$ is projective equivalent to none of (1)-(3) of Theorem 4. By Theorem $4, \mathcal{L}:=\mathcal{O}_{X}(\Delta-n) \otimes \omega_{X}^{\vee}$ has at most finite base locus for $\Delta:=\Delta\left(X, \mathcal{O}_{X}(1)\right)$, and hence we have (1) and (2).

\section{ACKNOWLEDGMENTS}

The author would like to thank Professors Euisung Park for calling attention to the result of Bo Ilic, Antonio Lanteri for suggesting Corollary 7.3, and Yoshiaki Fukuma for Remark 7.4.

\section{REFERENCES}

[1] Bo Ilic, Geometric properties of the double-point divisor, Trans. Amer. Math. Soc. 350 (1998), no. 4, 1643-1661, DOI 10.1090/S0002-9947-98-01928-X. MR.1422899 (98i:14010)

[2] Lawrence Ein and Robert Lazarsfeld, Syzygies and Koszul cohomology of smooth projective varieties of arbitrary dimension, Invent. Math. 111 (1993), no. 1, 51-67, DOI 10.1007/BF01231279. MR1193597 (93m:13006)

[3] David Eisenbud and Shiro Goto, Linear free resolutions and minimal multiplicity, J. Algebra 88 (1984), no. 1, 89-133, DOI 10.1016/0021-8693(84)90092-9. MR741934 (85f:13023)

[4] H. Flenner, L. O'Carroll, and W. Vogel, Joins and intersections, Springer Monographs in Mathematics, Springer-Verlag, Berlin, 1999. MR,1724388 (2001b:14010)

[5] Takao Fujita, Semipositive line bundles, J. Fac. Sci. Univ. Tokyo Sect. IA Math. 30 (1983), no. 2, 353-378. MR722501 (85f:32051)

[6] Takao Fujita, Classification theories of polarized varieties, London Mathematical Society Lecture Note Series, vol. 155, Cambridge University Press, Cambridge, 1990. MR.1162108 (93e:14009)

[7] Yoshiaki Fukuma, On the sectional geometric genus of quasi-polarized varieties. II, Manuscripta Math. 113 (2004), no. 2, 211-237, DOI 10.1007/s00229-003-0434-9. MR2128547 (2006b:14008)

[8] Mark Green and Robert Lazarsfeld, On the projective normality of complete linear series on an algebraic curve, Invent. Math. 83 (1985), no. 1, 73-90, DOI 10.1007/BF01388754. MR.813583 (87g:14022)

[9] L. Gruson, R. Lazarsfeld, and C. Peskine, On a theorem of Castelnuovo, and the equations defining space curves, Invent. Math. 72 (1983), no. 3, 491-506, DOI 10.1007/BF01398398. MR.704401 (85g:14033)

[10] Robin Hartshorne, Algebraic geometry, Springer-Verlag, New York, 1977. Graduate Texts in Mathematics, No. 52. MR0463157 (57 \#3116)

[11] Hajime Kaji, On the space curves with the same dual variety, J. Reine Angew. Math. 437 (1993), 1-11, DOI 10.1515/crll.1993.437.1. MR.1212250 (94a:14030)

[12] Robert Lazarsfeld, Positivity in algebraic geometry. I, Ergebnisse der Mathematik und ihrer Grenzgebiete. 3. Folge. A Series of Modern Surveys in Mathematics [Results in Mathematics and Related Areas. 3rd Series. A Series of Modern Surveys in Mathematics], vol. 48, Springer-Verlag, Berlin, 2004. Classical setting: line bundles and linear series. MR2095471 (2005k:14001a)

[13] David Mumford, Lectures on curves on an algebraic surface, with a section by G. M. Bergman, Annals of Mathematics Studies, No. 59, Princeton University Press, Princeton, N.J., 1966. MR0209285 (35 \#187) 
[14] Atsushi Noma, Multisecant subspaces to smooth projective varieties in arbitrary characteristic, Proc. Amer. Math. Soc. 137 (2009), no. 12, 3985-3990, DOI 10.1090/S0002-9939-0909977-8. MR2538558 (2010j:14095)

[15] Atsushi Noma, Hypersurfaces cutting out a projective variety, Trans. Amer. Math. Soc. 362 (2010), no. 9, 4481-4495, DOI 10.1090/S0002-9947-10-05054-3. MR.2645037(2011i:14088)

[16] Atsushi Noma, Projective varieties with nonbirational linear projections and applications, Preprint 2011.

[17] Jürgen Rathmann, The uniform position principle for curves in characteristic $p$, Math. Ann. 276 (1987), no. 4, 565-579, DOI 10.1007/BF01456986. MR879536 (89g:14026)

[18] Igor R. Shafarevich, Basic algebraic geometry. 1, 2nd ed., Springer-Verlag, Berlin, 1994. Varieties in projective space; translated from the 1988 Russian edition and with notes by Miles Reid. MR.1328833 (95m:14001)

[19] Oscar Zariski, The theorem of Riemann-Roch for high multiples of an effective divisor on an algebraic surface, Ann. of Math. (2) 76 (1962), 560-615. MR0141668 (25 \#5065)

Department of Mathematics, Yokohama National University, Yokohama 240-8501 JAPAN

E-mail address: noma@ynu.ac.jp 